\title{
1 Maltotriose consumption by hybrid Saccharomyces 2 pastorianus is heterotic and results from regulatory 3 cross-talk between parental sub-genomes
}

$4 \quad$ Heterotic origin of maltotriose consumption in Saccharomyces pastorianus

5 Nick Brouwers ${ }^{a}$, Anja Brickwedde ${ }^{a}$, Arthur R. Gorter de Vries ${ }^{a}$, Marcel van den Broek ${ }^{a}$, Susan M.

$6 \quad$ Weening ${ }^{a}$, Lieke van den Eijnden ${ }^{a}$, Jasper A. Diderich ${ }^{a}$, Feng-Yan Bai ${ }^{b}$, Jack T. Pronk ${ }^{a}$ and Jean-Marc G.

$7 \operatorname{Daran}^{\mathrm{a} *}$

${ }^{a}$ Department of Biotechnology, Delft University of Technology, Van der Maasweg 9, 2629 HZ Delft, The Netherlands.

bState Key Laboratory of Mycology, Institute of Microbiology, Chinese Academy of Sciences, Beijing, 100101, China.

* Corresponding author: Jean-Marc G. Daran, j.g.daran @tudelft.nl, Department of Biotechnology, Delft University of Technology, van der Maasweg 9, 2629 HZ Delft, The Netherlands

Nick Brouwers:

Anja Brickwedde:

Arthur R. Gorter de Vries:

Marcel van den Broek:

Susan M. Weening:

Lieke van den Eijnden:

Jasper A. Diderich:

Feng-Yan Bai:

Jack T. Pronk:

Jean-Marc G. Daran:
N.Brouwers-1@tudelft.nl

a.brickwedde@web.de

A.R.GorterdeVries@tudelft.nl

0000-0002-0841-6583

Marcel.vandenBroek@tudelft.nl

S.M.Weening@tudelft.nl

A.F.vandenEijnden@student.tudelft.nl

J.A.Diderich@tudelft.nl

baify@im.ac.cn

J.T.Pronk@tudelft.nl

0000-0002-5617-4611

J.G.Daran@tudelft.nl

0000-0003-3136-8193

Keywords: S. eubayanus, $\alpha$-oligo-glucoside metabolism, heterosis, domestication, experimental

evolution sequencing, brewing, hybridization. 


\section{Abstract}

S. pastorianus strains are hybrids of S. cerevisiae and S. eubayanus that have been domesticated for several centuries in lager-beer brewing environments. As sequences and structures of $S$. pastorianus genomes are being resolved, molecular mechanisms and evolutionary origin of several industrially relevant phenotypes remain unknown. This study investigates how maltotriose metabolism, a key feature in brewing, may have arisen in early S. eubayanus $\times$ S. cerevisiae hybrids. To address this question, we generated a near-complete genome assembly of Himalayan S. eubayanus strains of the Holarctic subclade. This group of strains have been proposed to be the origin of the S. eubayanus subgenome of current S. pastorianus strains. The Himalayan S. eubayanus genomes harbored several copies of a SeAGT1 $\alpha$-oligoglucoside transporter gene with high sequence identity to genes encountered in S. pastorianus. Although Himalayan S. eubayanus strains are unable to grown on maltose and maltotriose, their maltose-hydrolase and SEMALT1 and SEAGT1 maltose-transporter genes complemented the corresponding null mutants of $S$. cerevisiae. Expression, in a Himalayan $S$. eubayanus strain, of a functional S. cerevisiae maltose-metabolism regulator gene (MALx3) enabled growth on oligoglucosides. The hypothesis that the maltotriose-positive phenotype in S. pastorianus is a result of heterosis was experimentally tested by constructing a $S$. cerevisiae $\times$ S. eubayanus laboratory hybrid with a complement of maltose-metabolism genes that resembles that of current $S$. pastorianus strains. The ability of this hybrid to consume maltotriose in brewer's wort demonstrated regulatory cross talk between sub-genomes and thereby validated this hypothesis. These results provide experimental evidence of the evolutionary origin of an essential phenotype of lager-brewing strains and valuable knowledge for industrial exploitation of laboratory-made S. pastorianus-like hybrids.

\section{Importance}

S.pastorianus, a S.cerevisiae X S.eubayanus hybrid, is used for production of lager beer, the most produced alcoholic beverage worldwide It emerged by spontaneous hybridization and have colonized 
54 early lager-brewing processes. Despite accumulation and analysis of genome sequencing data of

55 S.pastorianus parental genomes, the genetic blueprint of industrially relevant phenotypes remains

56 unknown. Assimilation of wort abundant sugar maltotriose has been postulated to be inherited from

57 S.cerevisiae parent. Here, we demonstrate that although Asian S.eubayanus isolates harbor a

58 functional maltotriose transporter SeAGT1 gene, they are unable to grow on $\alpha$-oligoglucosides, but

59 expression of S. cerevisae regulator SCMAL13 was sufficient to restore growth on trisaccharides. We

60 hypothesized that S. pastorianus maltotriose phenotype results from regulatory interaction between

61 S.cerevisae maltose transcription activator and the promoter of SeAGT1. We experimentally

62 confirmed the heterotic nature of the phenotype and thus this results provide experimental evidence

63 of the evolutionary origin of an essential phenotype of lager-brewing strains. 


\section{Introduction}

66 Saccharomyces pastorianus is an interspecific hybrid of S. cerevisiae and S. eubayanus (1-4). S.

67 pastorianus strains are widely used for production of lager beer, which is currently the most produced alcoholic beverage worldwide. Lager brewing requires alcoholic fermentation at relatively low temperatures. S. pastorianus was hypothesized to have emerged by spontaneous hybridization and to have colonized early lager-brewing processes due to a combination of cold-tolerance inherited from S. eubayanus and superior fermentation kinetics inherited from S. cerevisiae (5-7). Lager beer is brewed from barley wort, whose sugar composition consists, by weight, of approximately $15 \%$ glucose, $60 \%$ maltose and $25 \%$ maltotriose (8). During wort fermentation, maltotriose is generally only utilized after glucose and maltose are depleted, while its consumption is also relatively slow and often incomplete (9-11).

Complete sugar utilization is desirable for lager beer fermentation to optimize concentrations of ethanol and flavor compounds and to avoid residual sweetness (12). While S. pastorianus and $S$. cerevisiae strains are capable of consuming maltotriose, none of the wild isolates of S. eubayanus characterized thus far have been shown to possess this trait $(6,13,14)$. These observations led to the hypothesis that the ability of $S$. pastorianus to ferment maltotriose was inherited from S. cerevisiae $(6,13,15-17)$.

The genetic information for maltose utilization is well conserved in Saccharomyces species and depends on three gene families. MALT genes encode plasma-membrane proton symporters with varying substrate specificities and affinities $(18,19)$, MALS genes encode $\alpha$-glucosidases that hydrolyze $\alpha$-oligoglucosides into glucose, while MALR genes encode a regulator required for transcriptional induction of MALT and MALS genes by maltose (20,21). In Saccharomyces species, maltose-utilization genes are generally organized in MAL loci. These loci contain a MALT gene (called SCMALX1 and SeMALTx in S. cerevisiae and S. eubayanus, respectively), a MALS gene referred to as 
90 of glucose and presence of maltose, the MalR regulator binds a bi-directional promoter, thereby

91 simultaneously activating expression of MALT and MALS genes (23).

The SCMAL1-SCMAL4 and SCMAL6 loci of S. cerevisiae as well as the SeMAL1-SeMAL4 loci of S. eubayanus are located in subtelomeric regions $(13,24-26)$. While all S. cerevisiae ScMalx1 transporters transport maltose, only SCMal11 is able to also transport maltotriose (9). SCMAL11 (also known as SCAGT1) shares only $57 \%$ nucleotide identity with other SCMALx1 genes (27). The four SeMALT (SeMALT1-4) genes identified in the genome of the Patagonian type strain FM1318/CBS 12357 of S. eubayanus were shown to encode functional maltose transporters, but none of these genes enabled maltotriose transport (13). While no clear SCAGT1 ortholog was found in S. eubayanus CBS $12357^{\top}$, such an ortholog was recently found in the genomes of two North American isolates assigned to the Holarctic subclade of S. eubayanus (14). functional in S. pastorianus (10). Instead, maltotriose consumption by S. pastorianus strains was attributed to SeAGT1 and SPMTY1/SPMTT1 genes (29-32). In S. pastorianus, SeAGT1 is located on S. eubayanus CHRXV and was therefore, already before the identification of an AGT1 ortholog in Holarctic S. eubayanus strains (14), assumed to originate from S. eubayanus (2). SpMTY1, also referred to as SPMTT1, is located on S. cerevisiae CHRVII and has less than $92 \%$ sequence identity with other Saccharomyces maltose transporters (30). However, SpMTY1 contains sequence patches with high similarity to maltose transporters from S. eubayanus and S. paradoxus (33). Recently, two

110 independent laboratory evolution studies with S. eubayanus demonstrated that recombination of 111 different SeMALT genes yielded chimeric, neo-functionalized genes that encoded maltotriose 112 transporters $(34,35)$. SPMTY1 may have resulted from successive introgressions of maltose113 transporter genes from S. cerevisiae, S. eubayanus and S. paradoxus. 
114 Recently made S. cerevisiae x S. eubayanus laboratory hybrids showed similar lager-brewing

115 performance as S. pastorianus strains, also with respect to maltotriose utilization $(6,16,17,36)$. In

116 these hybrids, maltotriose consumption depended on the presence of a functional ScAgt1

117 transporter encoded by the S. cerevisiae subgenome (37). However, in view of the non-functionality of SCAGT1 in current S. pastorianus strains, these laboratory hybrids did not fully recapitulate the genetic landscape of $S$. pastorianus with respect to maltotriose fermentation $(2,6,36)$.

to the $S$. eubayanus subgenome of $S$. pastorianus strains than that of the Patagonian type strain

122 CBS12357 might generate new insights into the evolution of maltotriose utilization in S. pastorianus.

123 To date, Himalayan S. eubayanus isolates show the highest sequence identity with the S. eubayanus 124 sub-genome of S. pastorianus, with up to $99.82 \%$ identity, as opposed to $99.56 \%$ for S. eubayanus

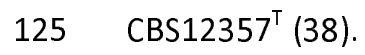

126 Here, we investigated if and how the genomes of Himalayan S. eubayanus strains could have 127 contributed to maltotriose utilization in the earliest hybrid ancestors of current S. pastorianus strains. To this end, we generated chromosome-level genome assemblies of these strains by longread DNA sequencing. Since the Himalayan strains were unable to utilize maltotriose, we functionally characterized the assembled $M A L$ genes and identified genetic determinants that prevented

131 maltotriose utilization. Subsequently, a laboratory hybrid of a representative Himalayan S. eubayanus

132 strain and a maltotriose-deficient ale strain of S. cerevisiae was generated to investigate the genetics

133 of maltotriose utilization in a hybrid context. We discuss the implications of the experimental results

134 for the proposed role and origin of SeAGT1 in S. pastorianus and for the potential of hybridization to 135 enable maltotriose consumption in novel Saccharomyces hybrids. 


\section{Results}

137 Sequencing of Himalayan S. eubayanus strains revealed variations of sub-telomeric regions and presence of novel putative maltose transporter genes.

It has been proposed that the $S$. eubayanus genetic pool of $S$. pastorianus was inherited from an ancestor of the Asian S. eubayanus lineage (38). With $99.82 \%$ identity, the Himalayan S. eubayanus strains CDFM21L.1 and ABFM5L.1 that belong to the Holarctic lineage (39), present the closest characterized relatives of the S. eubayanus ancestor of lager brewing yeasts. However, this distance was based on a limited sequencing space (38) and the analysis did not investigate the presence of specific S. eubayanus genetic markers found in S. pastorianus hybrids. Therefore, the genome of the Himalayan S. eubayanus strain CDFM21L.1 was sequenced with a combination of long-read and short-read techniques (Oxford Nanopore MinION and Illumina technologies, respectively) to generate a near-complete draft reference genome sequence. The resulting CDFM21L.1 genome assembly comprised 19 contigs, including its mitochondrial genome. All chromosomes were completely assembled from telomere to telomere, except for chromosome XII, which was fragmented into 3 contigs due to the repetitive rDNA region and manually assembled into a single scaffold. With a total size of $12,034,875 \mathrm{bp}$, this assembly represents the first near-complete draft genome of a S. eubayanus strain of the Holarctic clade (39).

Chromosome-level assemblies were hitherto only available for the Patagonia B-clade strain CBS $12357^{\top}(1,13)$. We identified three major structural differences in CDFM21L.1 relative to CBS12357 ${ }^{\top}$ using Mauve (40): (i) a paracentric inversion in the sub-telomeric region of chromosome VII, involving approximately $8 \mathrm{kbp}$, (ii) a translocation of approximately $12 \mathrm{kbp}$ from the left sub-telomeric region of chromosome VIII to the right sub-telomeric region of chromosome $\mathrm{VI}$, and (iii) a reciprocal translocation between approximately $20 \mathrm{kbp}$ from the right sub-telomeric region of chromosome $\mathrm{V}$ and approximately $60 \mathrm{kbp}$ from the center of chromosome XII (Figure 1A). All structural variation involved sub-telomeric regions, in accordance with their known relative instability (41-43). 
161 An alignment comparison of the CDFM21L.1 and CBS $12357^{\top}$ genomes with MUMmer revealed that

$162557 \mathrm{~kb}$ were unique to CDFM21L.1 and reciprocally $428 \mathrm{~kb}$ were unique to CBS $12357^{\top}$. Sequences

163 unique to CBS $12357^{\top}$ (3.6\% of its genome) and to CDFM21L.1 (4.6\% of its genome) were located

164 primarily in sub-telomeric regions and in repetitive regions, such as rDNA on chromosome XII (Figure

165 1B). Out of the 32 sub-telomeric regions, 23 exhibited absence of synteny. Conserved synteny was

166 observed for sub-telomeric regions on CHRIII (left), CHRIV (left and right), CHRVI (left), CHRIX (right),

167 CHRXI (right), CHRXII (right), CHRXIV (left) and CHRXV (right) (Supplementary File 1).

The $428 \mathrm{~kb}$ of sequence that were absent in the Himalyan S. eubayanus strain included 99 annotated

ORFs (Supplementary File 1). Of the 99 ORFs that were (partly) affected, 11 were completely absent

in CDFM21L.1, involving mostly genes implicated in iron transport facilitation (Supplementary File 1).

The $557 \mathrm{~kb}$ of sequence that was not present in CBS $12357^{\top}$ included 113 annotated ORFs

showed an overrepresentation of genes involved in transmembrane transport (Fishers exact test, P-

Of the 15 ORFs unique to CDFM21L.1, three were identical orthologs of S. cerevisiae MAL11/AGT1.

These three ORFs were found in the sub-telomeric regions of chromosomes VII, XIV and XV. Their of the SeMAL genes formed a canonical MAL locus in CDFM21L.1 (Figure 2). A systematic sequence inspection of these CDFM21L.1 SeMAL genes revealed mutations that prematurely interrupted the reading frames of SeMALR1 (CHRV), SeMALT2 (CHRXII) and SeMALT3 (CHRXII). 
187 SeMALT3 (CHRXII) were conserved. Two additional mutations were identified in one of the three

188 SeAGT1 genes. A nucleotide variation on position 53 and 939 (T instead of an A and A instead of a G))

189 resulted in a glycine to valine and arginine to lysine change, respectively.

Paradoxically, Himalayan S. eubayanus strains do not utilize maltose and maltotriose.

191

192

193

Identification of SeAGT1 in the two Himalayan S. eubayanus strains suggests an ability to not only grow on maltose but also on maltotriose. Strains from the Holarctic clade have previously been hypothesized to be the donor of the $S$. eubayanus sub-genome in $S$. pastorianus hybrids $(38,39)$. However, no physiological data regarding their ability to grow on the sugars present in wort are available. To assess their growth characteristics, the Asian S. eubayanus strains CDFM21L.1 and ABFM5L.1, the Patagonian S. eubayanus type strain CBS $12357^{\top}$ and the S. pastorianus strain CBS 1483 were grown on diluted industrial brewer's wort at $12{ }^{\circ} \mathrm{C}$. As reported previously, S. pastorianus strain CBS 1483 could utilize all three sugars but did not fully consume maltotriose (Figure 3) (11). Also in accordance with previous observations (6), CBS $12357^{\top}$ consumed glucose and maltose completely, but left maltotriose untouched. However, in marked contrast to S. eubayanus CBS $12357^{\top}$, neither CDFM21L.1 nor ABFM5L.1 consume maltose after growth on glucose. Moreover, like CBS $12357^{\top}$, maltotriose was not metabolized by these two S. eubayanus strains. While in CBS $12357^{\top}$ an ability to grow on maltose and an inability to grow on maltotriose could be readily attributed to its MAL genes complement, CDFM21L.1 and ABFM5L.1 failed to grow on maltose even though they appeared to contain complete genes encoding maltose (SeMALT1 and SeAGT1) and maltotriose (SeAGT1) transporters.

Growth defects on maltose and maltotriose are caused by deficiency of the regulatory SeMalR proteins in S. eubayanus CDFM21L.1.

The recent characterization of maltose metabolism in CBS $12357^{\top}$ showed that the coding regions of transcriptionally silent maltose-transporter genes in S. eubayanus can potentially encode functional 
212 proteins (13). The inability of the $S$. eubayanus Himalayan isolates to grow on $\alpha$-oligosaccharides

213 precluded direct testing of transporter-gene functionality by deletion studies. Instead, these genes

214 were expressed in S. cerevisiae IMZ616, which is devoid of all native maltose metabolism genes (44).

215 The CDFM21L.1 transporter genes SeMALT1, SeMALT2, SeMALT3 or SeAGT1 were integrated at the

216 SCSGA1 locus in IMZ616 along with the S. cerevisiae maltase gene SCMAL12 (13), yielding a series of

217 strains overexpressing a single transporter (IMX1702 (SeMALT1), IMX1704 (SeMALT2), IMX1706

218 (SeMALT3) and IMX1708 (SeAGT1)). These strains, as well as the negative and positive control strains

IMZ616 and IMX1365 (IMZ616 expressing SCAGT1 and SCMAL12), were grown on SM media

supplemented with either maltose or maltotriose. On maltose, not only the positive control strain

IMX1365, but also IMX1702 (SeMALT1) and IMX1708 (SeAGT1) were able to grow on maltose, maltose, both IMZ752 (SeMALS1) and IMZ753 (SeMALS2) grew and completely consumed maltose within $65 \mathrm{~h}$, demonstrating functionality of both hydrolase genes (Figure 4C). transcription factor encoded by MALx3 genes. Malx3 binds an activating site located in the

236 bidirectional promoters that control expression of $M A L x 2$ and $M A L x 1$ genes $(45,46)$. To test whether absence of maltose consumption in Himalayan S. eubayanus strains was caused by a lack 
transcriptional upregulation of SeMALT and SeMALS, the S. cerevisiae SCMAL13 gene was integrated at the SeSGA1 locus in S. eubayanus CDFM21L.1, under the control of a constitutive SCPGK1 promoter and SCTEF2 terminator. SCMAL13 expression in CDFM21L.1 enabled growth on maltose and maltotriose (Figure 5A), indicating that a lack of transcriptional upregulation was indeed the cause of the parental strain's inability to grow on these oligoglucosides. However, consumption of maltose and maltotriose was incomplete and consumed sugars were almost exclusively respired, as no ethanol was measured after $60 \mathrm{~h}$ of cultivation.

The possibility to grow an engineered variant of $S$. eubayanus CDFM21L.1 on $\alpha$-oligoglucosides offered an opportunity to study transporter function in its native context. Complementary functional characterization by gene deletion of SeMALT1 and SeAGT1 was performed using CRISPR-Cas9 genome editing method $(13,47)$. Deletion of SeMALT1 and SeAGT1 in CDFM21L.1 resulted in strains IMK820 and IMK823, respectively. Complete deletion of SeAGT1 required disruption of six alleles. To confirm the complete removal of all copies, the genome of IMK823 was sequenced. Mapping reads onto the reference S. eubayanus CDFM21L.1 genome assembly confirmed that all six alleles were removed simultaneously. Subsequently, the regulator expression cassette (SCPGK1 $1_{p}$ SCMAL13SCTEF2 $2_{t}$ ) was integrated in IMK820 and IMK823 at the SeSGA1 locus yielding strains IMX1939 and IMX1940, respectively (Figure 5B). The four deletion strains IMK820 (SemalT1A), IMK823 (Seagt1A),

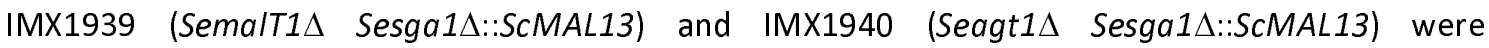
characterized on SMG, SMM or SMMt. All four strains were able to grow on glucose (Supplementary Figure 3). While strains IMK820, IMK823 and IMX1940 were unable to grow on maltose or

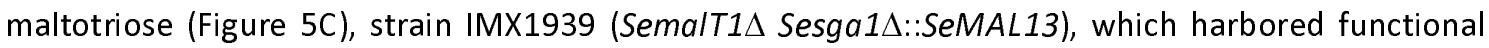
SeAGT1 copies, grew on maltose as well as on maltotriose. However, after $64 \mathrm{~h}$ of growth, these sugars were only partially consumed. Only $1.2 \mathrm{~g} \mathrm{~L}^{-1}$ ethanol was produced from maltose and no ethanol formation was observed during growth on maltotriose. The low ethanol concentration and the relatively high $\mathrm{OD}_{660 \mathrm{~nm}}$ suggest that, under the experimental conditions strain IMX1939 exhibited a Crabtree negative phenotype and exclusively respired maltotriose. S. eubayanus IMX1940 (Seagt1 $\Delta$ 
Sesga1 $\triangle:: S e M A L 13)$ did not consume maltotriose after $84 \mathrm{~h}$ of incubation. Moreover, despite the presence of SeMALT1, which encoded a functional maltose transporter upon expression in $S$. cerevisiae IMZ616, strain IMX1940 was also unable to consume maltose.

In addition to a functional Malx3 transcription factor, transcriptional activation of MAL genes also requires presence of a cis-regulatory motif in the promoter of regulated genes. Transcriptome analysis of S. eubayanus CBS $12357^{\top}$ recently showed that absence of a canonical cis-regulatory motif in SeMALT1 and SeMALT3 of S. eubayanus CBS $12357^{\top}$ caused a deficiency in their expression (13). To further explore regulation of SeMAL and SeAGT1 genes, we investigated the impact of carbon sources on genome-wide transcriptome and, specifically, on transcriptional activation of genes involved in maltose metabolism. Duplicate cultures of $S$. eubayanus strain IMX1765 (SCPGK1 $1_{\mathrm{p}^{-}}$ SCMAL13-SCTEF2 $2_{\mathrm{t}}$ ) were grown on SMG, SMM and SMMt at $20^{\circ} \mathrm{C}$ and sampled in mid-exponential phase. After mRNA isolation and processing, cDNA libraries and reads were assembled onto the newly annotated S. eubayanus CDFM21L.1 genome to calculate FPKM (fragments per kilobase of feature (gene) per million reads mapped) expression values. The heterologous regulator SCMAL13, expressed from the constitutive SCPGK1 promoter, displayed the same expression in glucose- and maltose-grown cultures. Although SCMAL13 was efficiently expressed on glucose, none of the nine $S$. eubayanus maltose genes (the three identical SeAGT1 copies being undistinguishable) were transcriptionally induced under these conditions (Figure 6), confirming that the hierarchical regulatory role of glucose catabolite repression $(45,48)$ also takes place in S. eubayanus. During growth on maltose, all nine genes were significantly upregulated relative to glucose-grown cultures but large variations in expression level were observed. The maltase genes SeMALS1 and SeMALS2 and the transporter gene SeAGT1 showed the highest upregulation, with fold changes of 148,161 and 2355 respectively. Although upregulated SeMALT1 displayed a fold change of 13, its normalized expression in maltose-grown cultures was $886-$ fold lower than that of SeAGT1. This weaker upregulation might explain why, despite the ability of its coding region to support synthesis of a 
transcriptome data also revealed that the absence of maltose induction in CDFM21L.1 was not associated with defective cis-regulatory elements in SeMALR promoter sequences since the regulator genes were properly activated, instead these results would suggest that the SeMaIR regulator are not functional.

\section{Hybridization of two maltotriose-deficient $S$. eubayanus and $S$. cerevisiae lineages results in} heterosis through regulatory crosstalk.

The genetic make-up of $S$. pastorianus lager-brewing yeasts strongly advocates that they originate from hybridization of $S$. cerevisiae and S. eubayanus parental lineages that were both unable to metabolize maltotriose (2). This hypothesis is consistent with the recurrent mutation in the $S$. cerevisiae AGT1 allele of $S$. pastorianus strains as well as with the inability of Himalayan strains of $S$. eubayanus to grow on these oligoglucosides.

Spores of the Himalayan S. eubayanus CDFM21L.1 were hybridized with S. cerevisiae CBC-1. This topfermenting S. cerevisiae is recommended for cask and bottle conditioning and unable to consume maltotriose (Lallemand, Montreal, Canada). Analysis of the CBC-1 assembly, obtained by a combination of long and short read sequencing, linked its maltotriose-negative phenotype to a total absence of the MAL11/AGT1 gene. The resulting laboratory interspecific hybrid HTSHO2O was characterized at $12{ }^{\circ} \mathrm{C}$ on synthetic wort, a defined medium whose composition resembles that of brewer's wort. While S. eubayanus CDFM21L.1 only consumed glucose and S. cerevisiae CBC-1 consumed glucose and maltose after $103 \mathrm{~h}$ (Figure 7A), the interspecific hybrid HTSH020 completely consumed glucose, maltose and partially consumed maltotriose after $105 \mathrm{~h}$, thus resembling characteristics of S. pastorianus strains (e.g. CBS1483 (11)). In addition to this gain of function, the hybrid HTSHO2O outperformed both its parents on maltose consumption, since it depleted this sugar in $70 \mathrm{~h}$ instead of $95 \mathrm{~h}$ for strain $\mathrm{CBC}-1$. Since $\mathrm{S}$. cerevisiae grows generally slower at $12{ }^{\circ} \mathrm{C}$, the experiments were also performed at $20^{\circ} \mathrm{C}$ where $\mathrm{HTSH} 020$ consumed all maltose $16 \mathrm{~h}$ earlier than CBC-1 (Supplementary Figure 4). 
315 Transcriptome analysis of the hybrid strain HTSHO2O grown on SM with different carbon sources

316 showed that SeAGT1 expression was repressed during growth on glucose, with a normalized

317 expression level of 7 FPKM (Figure 7B). When grown on SM maltose, SeAGT1, SeMALS1 and SeMALS2

318 were significantly induced, with fold increases of 816, 109 and 116, respectively (Figure 7B). Although

319 SeMALT1 and SeMALT2 were induced, these transporters do not contribute to maltose metabolism

320 due to truncation of their ORFs. These transcriptome data demonstrated that SeAGT1 and SeMALS

321 genes are induced by regulatory crosstalk between regulators encoded from the CBC-1 S. cerevisiae

322 sub-genome and maltotriose transporter genes harbored by the S. eubayanus genome. This laboratory hybridization experiment may be the closest reproduction yet of how, centuries ago, maltotriose-fermentation capacity arose in the first hybrid ancestor of S. pastorianus.

Discussion

The ability to consume maltose and maltotriose represents a key performance indicator of $S$. pastorianus lager-brewing strains (10). This study demonstrates how mating of S. cerevisiae and $S$. eubayanus strains that cannot themselves ferment maltotriose, can yield maltotriose-fermenting hybrids. This laboratory study illustrates how, centuries ago, maltotriose-fermentation capacity may have arisen in the first hybrid ancestor of $S$. pastorianus.

While the origin of the S. eubayanus parent of S. pastorianus strains is still under debate (49based on a limited sequencing space and was constrained by the quality of available sequence assemblies. Since an ortholog of SeAGT1 had previously only been found in the S. eubayanus subgenome of $S$. pastorianus strains, this finding revived the discussion on the geographical origin of the ancestral S. eubayanus parent (14). The high-quality, annotated genome assemblies of the Himalayan 
340 the previously proposed Asian origin of the S. eubayanus sub-genome of $S$. pastorianus $(14,38,39)$.

341 Next, genome-sequence comparison of the Patagonian B sub-clade S. eubayanus strain CBS12357

342 and the Holarctic sub-clade strain CDFM21L.1 revealed homoplasy of SeAGT1, probably reflecting

343 that these sub-clades evolved in different ecological niches.

344 The S. eubayanus wild stock whose genome sequence most closely corresponds to the $S$.

345 eubayanus sub-genome of $S$. pastorianus originates from the Tibetan plateau of the Himalaya (38).

346 However, the first S. cerevisiae $\times$ S. eubayanus hybrid, from which current lager yeasts evolved by

347 centuries of domestication, likely originates from a region between Bavaria and Bohemia in Central

348 Europe. So far, European S. eubayanus isolates have not been reported. This may indicate that the

349 original hybridization event occurred elsewhere or that the ancestral European lineage went extinct.

The recent detection, in a metagenomics analysis of samples from the Italian Alps, of ITS1 sequences

corresponding to S. eubayanus could indicate that a wild European lineage exists after all (52).

to an inability to transcriptionally upregulate maltose metabolism genes likely caused by loss of which a transporter (MALT) and a hydrolase (MALS) gene are expressed from the same bidirectional promoter and are located adjacent to a regulator gene (MALR) (21). In contrast, of the seven genomic regions harboring MAL genes in the two Asian S. eubayanus strains, none showed this canonical organization (Figure 2) and the subtelomeric regions carrying SeAGT1 did not harbour sequences similar to hydrolase or regulator genes. Subtelomeric regions harboring the other MAL genes indicated intensive reorganization as a result of recombination. In particular, subtelomeric 
genes from ancestral MAL1 and MAL2 loci over several chromosomes. A similar interpretation could explain the reorganization MAL3 on CHRXIII (Figure 2). Similar events may have contributed to loss of function of the MAL regulators (MaIR), as exemplified by the occurrence of a non-synonymous mutation in SeMALR1 resulting in loss of function. These rearrangements did, however, not inactivate the cis-regulatory sequences of the MAL genes, since complementation with a functional SCMAL13 allele caused induction of most SeMAL genes (Figure 6, Figure 7B) and, thereby, the heterotic maltotriose-positive phenotype of the hybrid strain HTSH020. Together with the high copy number of SeAGT1, this heterotic complementation may have been the main driver for colonization of low-temperature brewing processes by the early hybrid ancestors of current $S$. pastorianus strains. Recent work on adaptation to brewing environments of laboratory S. cerevisiae $\times$ S. eubayanus hybrids showed loss maltotriose utilization during serial transfer in wort (37). A similar loss of maltotriose utilization is frequently encountered in S. cerevisiae ale strains (54), as well as in some Saaz-type S. pastorianus strains (55). This is thus in contrast with retention of a maltotriose assimilation phenotype by Frohberg-type S. pastorianus strains. This may have been facilitated by the occurrence of multiple copies of the SeAGT1 gene in the S. eubayanus ancestor, which could act as a sequence buffer to counteracting adverse effects of gene copy loss. The recent release of the first long-read sequencing assembly of $S$. pastorianus enabled a precise chromosomal mapping of the maltose-metabolism genes (56) and showed that the Frohberg type S. pastorianus strains CBS 1483 harbored one copy of SeAGT1 on the S. eubayanus CHRXV section (as in CDFM21L.1) of the chimeric chromosome formed from SeCHRXV and SeCHRVIII (56).

Differential retention and loss of maltotriose consumption in S. pastorianus lineages may reflect different brewing process conditions during domestication. In modern brewing processes based on high-gravity wort, cell division is largely constrained to the glucose and maltose phases, which occur before depletion of nitrogen sources (57). It may be envisaged that, in early lager-brewing processes, unstandardized mashing processes generated wort with a higher maltotriose content, which would 
transfer on sugar mixtures, the selective advantage of consuming a specific sugar from a mixture correlates with the number of generations on that sugar during each cycle $(58,59)$. Such conditions would therefore have conferred a significant selective advantage to a maltotriose-assimilating $S$. cerevisiae $\times$ S. eubayanus hybrid, especially if, similar to current ale yeasts, the $S$. cerevisiae parent was unable to ferment maltotriose.

The heterotic phenotype that was reconstructed in the interspecies $S$. cerevisiae $x S$. eubayanus hybrid HTSO20 resulted from combination of dominant and recessive genetic variations from both parental genomes. S. eubayanus contributed the SeAGT1 gene and its functional cisregulatory sequences, but also harbored recessive mutations in MALR genes that allowed full expression of the heterotic phenotype. These mutations were complemented with a set of $S$. cerevisiae genes including a functional MALR and a non-functional SCAGT1 gene to match the mutations found in S. pastorianus (2). Although some S. pastorianus strains harbor an additional maltotriose transporter encoded by SPMTT1 (30), this gene was recently proposed to have emerged after the original hybridization event as a result of repeated recombination between MALT genes from both sub-genomes (33). understood and difficult to reproduce, that also might result from heterosis (37).

410 Laboratory-made S. cerevisiae x S. eubayanus hybrids hold great potential for brewing process 411 intensification and for increasing product diversity. In addition to increasing our understanding of the 412 evolutionary history of lager yeast genomes evolutionary, this study has implications for the design 413 of new hybrids. Hitherto, laboratory crosses of S. cerevisiae $\times$ S. eubayanus strains were designed 414 based on combination of dominant traits of the parental strains. Our results show that recessive 415 traits can be just as important as contributors to the genetic diversity of such hybrids. 


\section{Materials and methods}

\section{Strains and maintenance}

418 All strains used in this study are listed in Table 1. Stock cultures of S. eubayanus and S. cerevisiae 419 strains were grown in YPD (10 $\mathrm{g} \mathrm{L}^{-1}$ yeast extract, $20 \mathrm{~g} \mathrm{~L}^{-1}$ peptone and $20 \mathrm{~g} \mathrm{~L}^{-1}$ glucose $)$ until late 420 exponential phase, complemented with sterile glycerol to a final concentration of $30 \%(v / v)$ and 421 stored at $-80^{\circ} \mathrm{C}$ as $1 \mathrm{~mL}$ aliquots until further use.

\section{Media and cultivation}

S. eubayanus batch cultures were grown on synthetic medium (SM) containing $3.0 \mathrm{~g} \mathrm{~L}^{-1} \mathrm{KH}_{2} \mathrm{PO}_{4}, 5.0 \mathrm{~g}$

were sterilized by filtration and added to the sterile medium. Concentrated sugar solutions were autoclaved at $110{ }^{\circ} \mathrm{C}$ for 20 min or filter sterilized and added to the sterile flasks to give a final concentration of $20 \mathrm{~g} \mathrm{~L}^{-1}$ glucose (SMG), maltose (SMM) or maltotriose (SMMt). With the exception of IMZ752 and IMZ753, S. cerevisiae batch cultures were grown on SM supplemented with $150 \mathrm{mg} \mathrm{L}^{-1}$ uracil (62) to compensate for loss of plasmid pUDC156 that carried the Spcas9 endonuclease gene, and supplemented with $20 \mathrm{~g} \mathrm{~L}^{-1}$ glucose $\left(\mathrm{SM}_{\mathrm{u}} \mathrm{G}\right)$, maltose $\left(\mathrm{SM}_{\mathrm{u}} \mathrm{M}\right)$ or maltotriose $\left(\mathrm{SM}_{\mathrm{u}} \mathrm{Mt}\right)$. All batch cultures were grown in $250 \mathrm{~mL}$ shake flasks with a working volume of $50 \mathrm{~mL}$. The cultures were inoculated at an initial $O_{660 \mathrm{~nm}}$ of 0.1 and incubated under an air atmosphere and shaken at $200 \mathrm{rpm}$ and at $20{ }^{\circ} \mathrm{C}$ in a New Brunswick ${ }^{\mathrm{TM}}$ Innova44 incubator (Eppendorf Nederland B.V, Nijmegen, The Netherlands).

S. eubayanus strains transformed with plasmids pUDP052 (gRNA SeSGA1 $_{1}$ ), pUDP091 (gRNA SeMALT1 $_{\text {) and }}$

437 pUDP090 (gRNA $\left.A_{S E G T 1}\right)$ were selected on modified SMG medium $\left(\mathrm{SM}_{A c e} \mathrm{G}\right)$ in which $\left(\mathrm{NH}_{4}\right)_{2} \mathrm{SO}_{4}$ was replaced by $6.6 \mathrm{~g} \mathrm{~L}^{-1} \mathrm{~K}_{2} \mathrm{SO}_{4}$ and $10 \mathrm{mM}$ acetamide (63). SM-based solid media contained $2 \%$ Bacto Agar (BD Biosciences, Franklin Lakes, NJ). S. cerevisiae strains expressing either SeMALT, SeMALS or $440 S C M A L R$ were selected on $S_{\text {Ace }}$ G. For plasmid propagation, E. coli XL1-Blue-derived strains (Agilent

441 Technologies, Santa Clara, CA) were grown in Lysogeny Broth medium (LB, $10 \mathrm{~g} \mathrm{~L}^{-1}$ tryptone, $5 \mathrm{~g} \mathrm{~L}^{-1}$ 
442 yeast extract, $5 \mathrm{~g} \mathrm{~L}^{-1} \mathrm{NaCl}$ ) supplied with $100 \mathrm{mg} \mathrm{L}^{-1}$ ampicillin. Synthetic wort medium (SWM) for

443 growth studies contained $14.4 \mathrm{~g} \cdot \mathrm{L}^{-1}$ glucose, $2.3 \mathrm{~g} \cdot \mathrm{L}^{-1}$ fructose, $85.9 \mathrm{~g} \cdot \mathrm{L}^{-1} \mathrm{maltose}, 26.8 \mathrm{~g} \cdot \mathrm{L}^{-1}$

444 maltotriose, $5 \mathrm{~g} \cdot \mathrm{L}^{-1}\left(\mathrm{NH}_{4}\right)_{2} \mathrm{SO}_{4}, 3 \mathrm{~g} \cdot \mathrm{L}^{-1} \mathrm{KH}_{2} \mathrm{PO}_{4}, 0.5 \mathrm{~g} \cdot \mathrm{L}^{-1} \mathrm{MgSO}_{4} \cdot 7 \mathrm{H}_{2} \mathrm{O}, 1 \mathrm{~mL} \cdot \mathrm{L}^{-1}$ trace element solution, 1

$445 \mathrm{~mL} \cdot \mathrm{L}^{-1}$ vitamin solution, supplemented with the anaerobic growth factors ergosterol and Tween 80

$446\left(0.01 \mathrm{~g} \cdot \mathrm{L}^{-1}\right.$ and $0.42 \mathrm{~g} \cdot \mathrm{L}^{-1}$ respectively), as previously described (61).

447 Industrial wort (containing $14.4 \mathrm{~g} \mathrm{~L}^{-1}$ glucose, $85.9 \mathrm{~g} \mathrm{~L}^{-1}$ maltose, $26.8 \mathrm{~g} \mathrm{~L}^{-1}$ maltotriose, $2.3 \mathrm{~g} \mathrm{~L}^{-1}$

448 fructose and $269 \mathrm{mg} \mathrm{L^{-1 }}$ FAN) was provided by Heineken Supply Chain B.V. (Zoeterwoude, the

449 Netherlands). The wort was supplemented with $1.5 \mathrm{mg} \mathrm{L}^{-1}$ of $\mathrm{Zn}^{2+}$ by addition of $\mathrm{ZnSO}_{4} .7 \mathrm{H}_{2} \mathrm{O}$,

450 autoclaved for $30 \mathrm{~min}$ at 121 a , filtered using Nalgene $0.2 \mu \mathrm{m}$ SFCA bottle top filters (Thermo

451 Scientific) and diluted with sterile demineralized water. Sporulation medium consisted of $2 \%$ (w/v)

$452 \mathrm{KAC}$ in MilliQ water set to $\mathrm{pH} 7.0$ with $\mathrm{KOH}$, autoclaved at $121^{\circ} \mathrm{C}$ for $20 \mathrm{~min}$.

\section{Microaerobic growth experiments}

454 Microaerobic cultures were grown in 250-mL airlock-capped Neubor infusion bottles (38 mm neck,

455 Dijkstra, Lelystad, Netherlands) containing $200 \mathrm{~mL}$ three-fold diluted industrial wort supplemented

456 with $0.4 \mathrm{~mL} \mathrm{~L}^{-1}$ Pluronic antifoam (Sigma-Aldrich, St. Louis, MO). Bottle caps were equipped with a 0.5

$457 \mathrm{~mm} \times 16 \mathrm{~mm}$ Microlance needle (BD Biosciences) sealed with cotton to prevent pressure build-up.

458 Sampling was performed aseptically with $3.5 \mathrm{~mL}$ syringes using a $0.8 \mathrm{~mm} \times 50 \mathrm{~mm}$ Microlance needle

459 (BD Biosciences). Microaerobic cultures were inoculated at an $O_{660 n m}$ of 0.1 from stationary-phase

460 precultures in $50 \mathrm{~mL}$ Bio-One Cellstar Cellreactor tubes (Sigma-Aldrich) containing $30 \mathrm{~mL}$ of the same

461 medium, grown for 4 days at $12{ }^{\circ} \mathrm{C}$. Bottles were incubated at $12{ }^{\circ} \mathrm{C}$ and shaken at $200 \mathrm{rpm}$ in a $\mathrm{New}$

462 Brunswick Innova43/43R shaker (Eppendorf Nederland B.V.). At regular intervals, $3.5 \mathrm{~mL}$ samples

463 were collected in 24 deep-well plates (EnzyScreen BV, Heemstede, Netherlands) using a LiHa liquid

464 handler (Tecan, Männedorf, Switzerland) to measure OD $660 \mathrm{~nm}$ and external metabolites. $30 \mu \mathrm{L}$ of each

465 sample was diluted 5-fold in demineralized water in a 96 well plate and OD $_{660 n m}$ was measured with a

466 Magellan Infinite 200 PRO spectrophotometer (Tecan). From the remaining sample, $150 \mu \mathrm{L}$ was 
467 vacuum filter sterilized using $0.2 \mu \mathrm{m}$ Multiscreen filter plates (Merck, Darmstadt, Germany) for HPLC

468 measurements.

\section{Analytical methods}

470 Optical densities of yeast cultures were measured with a Libra S11 spectrophotometer (Biochrom,

471 Cambridge, United Kingdom) at a wavelength of $660 \mathrm{~nm}$. Biomass dry weight was measured by

472 filtering $10-\mathrm{mL}$ culture samples over pre-weighed nitrocellulose filters with a pore size of $0.45 \mu \mathrm{m}$.

473 Filters were washed with $10 \mathrm{~mL}$ water, dried in a microwave oven ( $20 \mathrm{~min}$ at $350 \mathrm{~W}$ ) and reweighed.

474 Sugars were measured using a high pressure liquid chromatography Agilent Infinity 1260 series

475 (Agilent Technologies) using a Bio-Rad Aminex HPX-87H column at $65^{\circ} \mathrm{C}$ with $5 \mathrm{mM}$ sulfuric acid at a

476 flow rate of $0.8 \mathrm{~mL} \mathrm{~min}{ }^{-1}$. Compounds were measured using a RID at $35^{\circ} \mathrm{C}$. Samples were centrifuged

477 at $13,000 \mathrm{~g}$ for $5 \mathrm{~min}$ to collect supernatant or $0.2 \mu \mathrm{m}$ filter-sterilized before analysis.

478

479

480

481

482

484

485

486

487

488

489

490

\section{Plasmid construction}

Plasmids used and constructed in this study are listed in Table 2, oligonucleotide primers used in this study are listed in Supplementary Table 1. Coding regions of SeMALT1, SeMALT2, SeMALT3 and SeAGT1 were amplified from CDFM21L.1 genomic DNA with Phusion High-Fidelity DNA polymerase (Thermo Scientific), according to the supplier's instructions with primers pairs 12355/12356, $12357 / 12358,12359 / 12360$ and $12361 / 12362$, respectively. The coding sequence of ScMAL31 was amplified from CEN.PK113-7D genomic DNA with Phusion High-Fidelity DNA polymerase (Thermo Scientific), according to the supplier's instructions with primer pairs 9942/9943. Each primer carried a 40 bp extension complementary to the plasmid backbone of p426-TEF-amdS (64), which was PCR amplified using Phusion High-Fidelity DNA polymerase (Thermo Scientific) and primers 7812/5921. Each transporter fragment was assembled with the p426-TEF-amdS backbone fragment using NEBuilder HiFi DNA Assembly (New England Biolabs, Ipswich, MA), resulting in plasmids pUD444 (SCMAL31), pUD794 (SeMALT1), pUD795 (SeMALT2), pUD796 (SeMALT3) and pUD797 (SeAGT1). All 
491 plasmids were verified for correct assembly by Sanger sequencing (Baseclear, Leiden, The

492 Netherlands).

493

494

495

496

497

498

499

500

501

502

503

504

505

506

507

508

509

510

511

512

513

514

515

SeMALS1 and SeMALS2 were amplified from CDFM21L.1 genomic DNA with Phusion High-Fidelity DNA polymerase (Thermo Scientific), with primers pairs 14451/14453 and 14452/14453, respectively. Each primer pair carried a 30 bp extension complimentary to the plasmid backbone of pUDE044 (65) which was PCR amplified using Phusion High-Fidelity DNA polymerase (Thermo Scientific) and primers 14449/14450. Resulting amplicons were assembled using NEBuilder HiFi DNA Assembly (New England Biolabs), resulting in plasmids pUDE843 (SeMALS1) and pUDE844 (SeMALS2) that were verified by Sanger sequencing (Baseclear).

S. cerevisiae SCMAL13, the SCPGK1 promoter and the SCTEF2 terminator were amplified from CEN.PK113-7D genomic DNA with Phusion High-Fidelity DNA polymerase (Thermo Scientific), with primer pairs 12915/12916, 9421/9422 and 10884/10885, respectively. Fragments were gel purified and used with pYTK074, pYTK082 and pYTK083 in Golden Gate assembly according to the yeast toolkit protocol (66) resulting in pUDE780, which was verified by Sanger sequencing (Baseclear).

Guide-RNA (gRNA) sequences for deletion of SeMALT1 and SeAGT1 in CDFM21L.1 were designed as described previously (47). The DNA sequences encoding these gRNAs were synthesized at GeneArt (Thermo Scientific) and were delivered in pUD631 and pUD634, respectively. The gRNA spacer sequences (SeMALT1 5' CCCCGATATTCTTTACACTA 3', SeAGT1 5'- AGCTTTGCGAAAATATCCAA -3') and the structural gRNA sequence were flanked at their $5^{\prime}$ ends by the Hammerhead ribozyme (HH) and at their $3^{\prime}$ ends by the Hepatitis Delta Virus ribozyme (HDV) (67). The HH-gRNA-HDV fragment was flanked on both ends with a Bsal site for further cloning $(47,68)$. Plasmids pUDP091 (gRNA

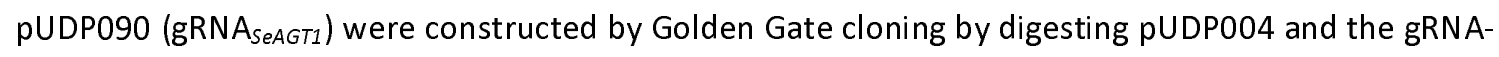
carrying plasmid (pUD631 and pUD634, respectively) using Bsal and ligating with T4 ligase (69). Correct assembly was verified by restriction analysis with Pdml (Thermo Scientific) and Sanger sequencing (Baseclear). 


\section{Strain construction}

517 S. cerevisiae IMZ616, which cannot grow on $\alpha$-glucosides (44), was used as a host to test functionality

518 of individual S. eubayanus (putative) maltose transporter genes (13). S. cerevisiae IMX1702 was 519 constructed by integrating $S C T D H 3_{p}-S C M A L 12-S C A D H 1_{t}$ and $S C T E F 1_{p}-S e M A L T 1-S C C Y C 1_{t}$ at the $S C S G A 1$

520 locus of strain IMZ616. A fragment containing the $S C T D H 3_{p}-S C M A L 12-S C A D H 1_{t}$ transcriptional unit 521 was PCR amplified using Phusion High-Fidelity DNA polymerase (Thermo Scientific) from pUDE044 with primers 9596/9355, which included a 5' extension homologous to the upstream region of the SCSGA1 locus and an extension homologous to the co-transformed transporter fragment, respectively. The DNA fragment carrying the $S$. eubayanus SeMALT1 maltose symporter $\left(S C T E F 1_{p^{-}}\right.$ SeMALT1-SCCYC1 $1_{t}$ ) was PCR amplified from pUD794 using primers $9036 / 9039$, which included a $5^{\prime}$ extension homologous to the co-transformed transporter fragment and an extension homologous to the downstream region of the SCSGA1 locus, respectively. To facilitate integration in strain IMZ616, the two PCR fragments were co-transformed with plasmid pUDR119 (amdS), which expressed a gRNA targeting SCSGA1 (spacer sequence: 5'-ATTGACCACTGGAATTCTTC-3') (70). The plasmid and repair 530 fragments were transformed using the LiAc yeast transformation protocol (71) and transformed cells

531 were plated on $\mathrm{SM}_{\mathrm{Ace}} \mathrm{G}$. Correct integration was verified by diagnostic PCR with primers pairs 532 4226/4224. Strains S. cerevisiae IMX1704, IMX1706 and IMX1708 were constructed following the 533 same principle, but instead of using pUD794 to generate the transporter fragment, pUD795, pUD796 534 and pUD797 were used to PCR amplify SCTEF1 $1_{p}-S e M A L T 2-S C C Y C 1_{t}, S C T E F 1_{p}-S e M A L T 3-S C C Y C 1_{t}$ and 535 SCTEF1 $1_{p}-S_{e A G T 1-S C C Y C 1_{t}}$ respectively. IMX1313 was constructed in a similar way using only SCTEF1 $p_{p^{-}}$ 536 SCMAL31-SCCYC1 $1_{t}$ amplified with primer pair 9036/11018 which contain 5'and $3^{\prime}$ extensions 537 homologous to the upstream and downstream region of the SCSGA1 locus. Correct integration was 538 verified by diagnostic PCR with primer pair 4226/4224 (Supplementary Figure 1). All PCR-amplified gene sequences were Sanger sequenced (BaseClear). IMX1313 was grown on YPD to loose pUDR119

540 (URA3) and pUDC156 (amdS). An isolate unable to grown on SMG without uracil and with acetamide 
541 was selected and named IMX1313 $\Delta$. This strain was able to grow on SMG supplemented with $150 \mathrm{mg}$

$542 \quad \mathrm{~L}^{-1}$ uracil.

543 To assess functionality of CDFM21L.1 SeMALS1, IMX1313A was transformed with 100 ng pUDE843

$544\left(S c T D H 3_{p}-S e M A L S 1-S C A D H 1_{t}\right)$ by electroporation (47), resulting in strain IMZ752. Transformants were

545 selected on SMG plates after 5 days of incubation at $20^{\circ} \mathrm{C}$ and validated by PCR (DreamTaq

546 polymerase, Thermo Scientific) using primer pair 14454/14455 (Supplementary Figure 1). Similarly,

547 functionality of the SeMALS2 maltase gene of CDFM21L.1 was assessed by transforming IMX1313

548 with pUDE844 $\left(S C T D H 3_{\mathrm{pr}}-\right.$ SeMALS2-SCADH1 $\left.1_{\text {ter }}\right)$, resulting in strain IMZ753.

549 S. eubayanus IMK820 (SemalT1A) was constructed by transforming CDFM21L.1 with $200 \mathrm{ng}$ of 550 pUDP091 and $1 \mu \mathrm{g}$ of 120 bp repair fragment obtained by mixing an equimolar amount of primers 551 12442/12443, as previously described (47). As a control, the same transformation was performed 552 without including the repair DNA fragment. Transformants were selected on SM Ace plates. S. 553 eubayanus IMK823 (Seagt1A) was constructed similarly, using pUDP090 and primer pair 554 11320/11321. Deletion of SemalT1 was verified by PCR with primer pair 11671/11672 and Sanger 555 sequencing. The Seagt1 deletion was verified by PCR using primer pair 12273/12274, and by Illumina 556 whole-genome sequencing and read alignment to the reference genome of CDFM21L.1 (Bioproject 557 accession number PRJNA528469).

558 Strains IMX1765, IMX1939 and IMX1940 were constructed by inserting ScPGK1 $1_{p}-S C M A L 13-S c T E F 2_{t}$ at 559 the SeSGA1 locus of CDFM21L.1, IMK820 and IMK823, respectively. A repair fragment containing SCPGK1 $1_{p}$ SCMAL13-SCTEF2 $t$ was amplified from pUDE780 with primer pair $12917 / 12918$. Strains

561 CDFML21L.1, IMK820 and IMK823 were transformed by electroporation by addition of $350 \mathrm{ng}$ of 562 repair fragment and $560 \mathrm{ng}$ pUDP052 (amdS) into the cells as previously described (47). 563 Transformants were plated on $\mathrm{SM}_{\text {Ace }} \mathrm{G}$ and incubated at $20{ }^{\circ} \mathrm{C}$. IMX1762 was constructed similarly 564 using a repair fragment with $S C T D H 3_{p}-S C M A L 12-S C A D H 1_{t}$ amplified from pUDE044 with primer pair 565 12319/12320. Strains were verified by PCR using primer pair 12635/12636 and Sanger sequencing. 


\section{Hybrid Construction}

567 The S. cerevisiae X S. eubayanus hybrid HTSH02O was constructed by spore-to-spore mating. The S.

568 eubayanus strain CDFM21L.1 and the S. cerevisiae strain CBC-1 were grown in $20 \mathrm{~mL}$ YPD at $20^{\circ} \mathrm{C}$

569 until late exponential phase. Cells were centrifuged for $5 \mathrm{~min}$ at $1000 \mathrm{~g}$ and washed twice in

570 demineralized water. Cells were re-suspended in $20 \mathrm{~mL}$ sporulation medium and incubated for $64 \mathrm{~h}$

571 at $20^{\circ} \mathrm{C}$. Presence of spores was verified by microscopy. Asci were harvested by centrifugation for 5

$572 \mathrm{~min}$ at $1000 \mathrm{~g}$, and washed with demineralized water, resuspended in $100 \mu \mathrm{L}$ demineralized water

573 containing $100 \mathrm{U} / \mathrm{mL}$ of Zymolyase (MP Bio, Santa Ana, CA) and incubated for $10 \mathrm{~min}$ at $30^{\circ} \mathrm{C}$. Spores

574 were washed and plated on the edge of a YPD agar plate. Spores from the two strains were brought

575 in contact with each other with an MSM System 400 micromanipulator (Singer Instruments, Watchet,

576 United Kingdom). Zygote formation was observed after 6-8 h. Emerging colonies were re-streaked

577 twice on SM $2 \%$ maltose at $12{ }^{\circ} \mathrm{C}$. Successful hybridization was verified by multiplex PCR using

578 DreamTaq DNA polymerase (Thermo Scientific), by amplifying the S. cerevisiae specific MEX67 gene

579 with primer pairs $8570 / 8571$ and by amplifying the S. eubayanus specific gene SeFSY1 with primers

$5808572 / 8573$ (Supplementary Figure 2), as previously described (72).

581 Illumina sequencing

582 Genomic DNA of $S$. eubayanus strains CDFM21L.1 and ABFM5L.1, S. cerevisiae strain CBC-1 and S.

583 cerevisiae $\times$ S. eubayanus strain HTSH020 was isolated as previously described (4). Paired-end

584 sequencing ( $2 \times 150$ bp) was performed on a 350 bp PCR-free insert library using Illumina HiSeq2500

585 (San Diego, CA) by Novogene(HK) Company Ltd (Hong Kong, China). Genomic DNA of the strains CBC-

5861 and HTSH020 was sequenced in house on a MiSeq sequencer (Illumina) with 300 bp paired-end

587 reads using PCR-free library preparation. Sequence data are available at NCBI under Bioproject

588 accession number PRJNA528469. 


\section{MinION long read sequencing}

590 For long-read sequencing, a 1D sequencing library (SQK-LSK108) was prepared for CDFM21L.1 and

591 CBC-1 and loaded onto an FLO-MIN106 (R9.4) flow cell, connected to a MinION Mk1B unit (Oxford

592 Nanopore Technology, Oxford, United Kingdom), according to the manufacturer's instructions.

593 MinKNOW software (version 1.5.12; Oxford Nanopore Technology) was used for quality control of

594 active pores and for sequencing. Raw files generated by MinKNOW were base-called using Albacore

595 (version 1.1.0; Oxford Nanopore Technology). Reads with a minimum length of 1000 bp were

596 extracted in fastq format. For CDFM21L.1, 3.26 Gb of sequence with an average read length of 8.07

$597 \mathrm{~kb}$ was obtained and for $\mathrm{CBC}-13.04 \mathrm{~Gb}$ sequence with an average read length of $7.27 \mathrm{~kb}$. Sequencing

598 data are available at NCBI under Bioproject accession number PRJNA528469.

De novo assembly

600 De novo assembly of the Oxford Nanopore MinION dataset was performed using Canu (v1.4, setting:

601 genomesize $=12 \mathrm{~m}$ ) (73). Assembly correctness was assessed using Pilon (74) and further corrected by

602 "polishing" of sequencing/assembly errors by aligning Illumina reads with BWA (75) using correction

603 of only SNPs and short indels (-fix bases parameter). For HTSH020, an artificial reference genome

604 was made by combining the assembly of CBC-1 and CDFM21L.1. The genome assemblies were annotated using the MAKER2 annotation pipeline (version 2.31.9) (76), using SNAP (version 2013-11-

29) (77) and Augustus (version 3.2.3) (78) as ab initio gene predictors. S. cerevisiae S288C EST and

607 protein sequences were obtained from SGD (Saccharomyces Genome Database, assembly (BLAST version 2.2.28+) (79). Predicted translated protein sequences of the final gene model were aligned to the S. cerevisiae S288C protein Swiss-Prot database using BLASTP

611 (http://www.uniprot.org/). Custom-made Perl scripts were used to map systematic names to the 612 annotated gene names (Supplementary Table 2). Error rates in nanopore-sequencing data were 613 estimated from the q score (Phred scaled) per read, as calculated by the base caller Albacore (version 614 1.1.0) (Oxford Nanopore Technology). Average $q$ score was used to calculate the error $P=10^{q / 10}$. 


\section{RNA isolation}

617 CDFM21L.1, IMX1765, IMX1939 and HTS020 were grown in SMG, SMM and SMMt until midexponential phase $\left(\mathrm{OD}_{660 \mathrm{~nm}}\right.$ of 12 for $\mathrm{SMG} / \mathrm{SMM}$ and of $\mathrm{OD}_{660 \mathrm{~nm}} 15$ for $\left.\mathrm{SMMt}\right)$. Culture samples corresponding to ca. $200 \mathrm{mg}$ of biomass dry weight were directly quenched in liquid nitrogen. The samples were processed and total RNA extracted as previously described (80). Prior to cDNA synthesis, purity, concentration and integrity of the RNA in the samples was assessed with Nanodrop

622 (Thermo Scientific), Qubit (Thermo Scientific) and Tapestation 220 with RNA Screen Tape (Agilent 623 Technologies), respectively, according the manufacturers' recommendations. cDNA libraries were prepared using the TruSeq RNA V2 kit (IIlumina). Paired-end sequencing ( 2 X $150 \mathrm{bp}$ ) was performed on a 300 bp PCR-free insert library on a HISeq 2500 (Illumina) at Novogene (HK) Company Ltd (Hong Kong, China). Duplicate biological samples were processed, generating an average sequence quantity of $23.7 \mathrm{M}$ reads per sample. Reads were aligned to the CDFM21L.1 reference assembly (GEO (https://www.ncbi.nlm.nih.gov/geo/) accession number GSE133146) using a two-pass STAR (81) procedure. In the first pass, splice junctions were assembled and used to inform the second round of alignments. Introns between 15 and 4000 bp were allowed, and soft clipping was disabled to prevent low-quality reads from being spuriously aligned. Ambiguously-mapped reads were removed from the dataset. Expression level for each transcript were quantified using htseq-count (82) in union mode. Fragments per kilo-base of feature (gene) per million reads mapped (FPKM) values were calculated

634 by "Applying the fpkm method" from the edgeR package $(83,84)$. Differential expression analysis was 635 performed using DESeq (85). 


\section{Accession Numbers}

638 The sequencing data were deposited at NCBI (https://www.ncbi.nlm.nih.gov/) under the Bioproject

639 PRJNA528469 and the transcriptomics data were deposited at GEO (Genome Expression Omnibus

640 https://www.ncbi.nIm.nih.gov/geo/) under accession number GSE133146.

641

\section{Supplementary Data}

643 Supplementary Data are available

644

\section{Authors contributions}

646 JMD conceived the study and designed the experiments. NB, AB, LvbE, ARGdV, SMW and JD

647 performed the experimental work. NB and MvdB performed bioinformatics analysis. NB, AB, FYB, JTP and JMGD supervised the study and wrote the manuscript. All authors read and approved the final manuscript.

\section{Acknowledgements}

651 We thank Flip de Groot for his technical assistance in gene editing in S. eubayanus, Niels G.A.

652 Kuijpers, Viktor M. Boer and Jan-Maarten Geertman for their critical input.

\section{Funding}

655 This work was supported by the BE-Basic R\&D Program (http://www.be-basic.org/), which was 656 granted an FES subsidy from the Dutch Ministry of Economic Affairs, Agriculture and Innovation 657 (EL\&I).

658 
659

660

661

662

663

664

665

666

667

668

669

670

671

672

673

674

675

676

677

678

679

680

681

682

683

684

685

686

687

688

689

690

691

692

693

694

695

696

697

698

699

700

701

702

703

704

705

\section{Conflict of Interest}

The authors declare no conflict of interest.

\section{References}

1. Libkind D, Hittinger CT, Valério E, Gonçalves C, Dover J, Johnston $M$, Gonçalves $P$, Sampaio JP. 2011. Microbe domestication and the identification of the wild genetic stock of lagerbrewing yeast. Proc Natl Acad Sci U S A:201105430.

2. Nakao Y, Kanamori T, Itoh T, Kodama Y, Rainieri S, Nakamura N, Shimonaga T, Hattori M, Ashikari T. 2009. Genome sequence of the lager brewing yeast, an interspecies hybrid. DNA Res 16:115-129.

3. Walther A, Hesselbart A, Wendland J. 2014. Genome sequence of Saccharomyces carlsbergensis, the world's first pure culture lager yeast. G3 (Bethesda) 4:783-793.

4. Van den Broek M, Bolat I, Nijkamp JF, Ramos E, Luttik MAH, Koopman F, Geertman JM, De Ridder D, Pronk JT, Daran J-MG. 2015. Chromosomal copy number variation in Saccharomyces pastorianus evidence for extensive genome dynamics in industrial lager brewing strains. Appl Environ Microbiol:AEM. 01263-01215.

5. Krogerus K, Magalhães F, Vidgren V, Gibson B. 2015. New lager yeast strains generated by interspecific hybridization. J Ind Microbiol Biotechnol 42:769-778.

6. Hebly M, Brickwedde A, Bolat I, Driessen MR, de Hulster EA, van den Broek M, Pronk JT, Geertman J-M, Daran J-MG, Daran-Lapujade P. 2015. S. cerevisiae $\times$ S. eubayanus interspecific hybrid, the best of both worlds and beyond. FEMS Yeast Res 15.

7. Krogerus K, Magalhães F, Vidgren V, Gibson B. 2017. Novel brewing yeast hybrids: creation and application. Appl Environ Microbiol 101:65-78.

8. Zastrow C, Hollatz C, De Araujo P, Stambuk B. 2001. Maltotriose fermentation by Saccharomyces cerevisiae. J Ind Microbiol Biotechnol 27:34-38.

9. Alves SL, Herberts RA, Hollatz C, Trichez D, Miletti LC, De Araujo PS, Stambuk BU. 2008. Molecular analysis of maltotriose active transport and fermentation by Saccharomyces cerevisiae reveals a determinant role for the AGT1 permease. Appl Environ Microbiol 74:1494-1501.

10. Vidgren V, Huuskonen A, Virtanen H, Ruohonen L, Londesborough J. 2009. Improved fermentation performance of a lager yeast after repair of its AGT1 maltose and maltotriose transporter genes. Appl Environ Microbiol 75:2333-2345.

11. Brickwedde A, van den Broek M, Geertman J-MA, Magalhães F, Kuijpers NG, Gibson B, Pronk JT, Daran J-MG. 2017. Evolutionary engineering in chemostat cultures for improved maltotriose fermentation kinetics in Saccharomyces pastorianus lager brewing yeast. Front Microbiol 8:1690.

12. Zheng X, D'Amore T, Russell I, Stewart G. 1994. Factors influencing maltotriose utilization during brewery wort fermentations. J Am Soc Brew Chem 52:41-47.

13. Brickwedde A, Brouwers N, van den Broek M, Gallego Murillo JS, Fraiture JL, Pronk JT, Daran J-MG. 2018. Structural, physiological and regulatory analysis of maltose transporter genes in Saccharomyces eubayanus CBS 12357T. Front Microbiol 9:1786.

14. Baker EP, Hittinger CT. 2018. Evolution of a novel chimeric maltotriose transporter in Saccharomyces eubayanus from parent proteins unable to perform this function. bioRxiv doi:10.1101/431171.

15. Krogerus K, Magalhães F, Vidgren V, Gibson B. 2017. Novel brewing yeast hybrids: creation and application. Appl Microbiol Biotechnol 101:65-78.

16. Krogerus K, Magalhães F, Vidgren V, Gibson B. 2015. New lager yeast strains generated by interspecific hybridization. Journal of industrial microbiology \& biotechnology 42:769-778. 
17. Nikulin J, Krogerus K, Gibson B. 2018. Alternative Saccharomyces interspecies hybrid combinations and their potential for low-temperature wort fermentation. Yeast (Chichester, England) 35:113-127.

18. Cheng Q, Michels CA. 1991. MAL11 and MAL61 encode the inducible high-affinity maltose transporter of Saccharomyces cerevisiae. J Bacteriol 173:1817-1820.

19. Vidgren V, Multanen JP, Ruohonen L, Londesborough J. 2010. The temperature dependence of maltose transport in ale and lager strains of brewer's yeast. FEMS Yeast Res 10:402-411.

20. Naumov GI, Naumova ES, Michels C. 1994. Genetic variation of the repeated MAL loci in natural populations of Saccharomyces cerevisiae and Saccharomyces paradoxus. Genetics 136:803-812.

21. Charron MJ, Read E, Haut SR, Michels CA. 1989. Molecular evolution of the telomereassociated MAL loci of Saccharomyces. Genetics 122:307-316.

22. Hayford A, Jespersen L. 1999. Characterization of Saccharomyces cerevisiae strains from spontaneously fermented maize dough by profiles of assimilation, chromosome polymorphism, PCR and MAL genotyping. Journal of applied microbiology 86:284-294.

23. Bell PJ, Higgins VJ, Dawes IW, Bissinger PH. 1997. Tandemly repeated 147 bp elements cause structural and functional variation in divergent MAL promoters of Saccharomyces cerevisiae. Yeast 13:1135-1144.

24. Barnett JA. 1976. The Utilization of Sugars by Yeasts1, p 125-234, Advances in carbohydrate chemistry and biochemistry, vol 32. Elsevier.

25. Salazar AN, Gorter de Vries AR, van den Broek $\mathbf{M}$, Wijsman $\mathbf{M}$, de la Torre Cortés $\mathbf{P}$, Brickwedde A, Brouwers N, Daran J-MG, Abeel T. 2017. Nanopore sequencing enables nearcomplete de novo assembly of Saccharomyces cerevisiae reference strain CEN. PK113-7D. FEMS Yeast Res 17.

26. Baker E, Wang B, Bellora N, Peris D, Hulfachor AB, Koshalek JA, Adams M, Libkind D, Hittinger CT. 2015. The genome sequence of Saccharomyces eubayanus and the domestication of lager-brewing yeasts. Mol Biol Evol 32:2818-2831.

27. Han EK, Cotty F, Sottas C, Jiang H, Michels CA. 1995. Characterization of AGT1 encoding a general a-glucoside transporter from Saccharomyces. Mol Microbiol 17:1093-1107.

28. Vidgren V, Gibson B. 2018. Trans-regulation and localization of orthologous maltose transporters in the interspecies lager yeast hybrid. FEMS yeast research 18:foy065.

29. Salema-Oom M, Pinto VV, Gonçalves P, Spencer-Martins I. 2005. Maltotriose utilization by industrial Saccharomyces strains: characterization of a new member of the $\alpha$-glucoside transporter family. Appl Environ Microbiol 71:5044-5049.

30. Dietvorst J, Londesborough J, Steensma H. 2005. Maltotriose utilization in lager yeast strains: MTT1 encodes a maltotriose transporter. Yeast 22:775-788.

31. Cousseau F, Alves Jr S, Trichez D, Stambuk B. 2013. Characterization of maltotriose transporters from the Saccharomyces eubayanus subgenome of the hybrid Saccharomyces pastorianus lager brewing yeast strain Weihenstephan 34/70. Lett Appl Microbiol 56:21-29.

32. Nguyen H-V, Legras J-L, Neuvéglise C, Gaillardin C. 2011. Deciphering the hybridisation history leading to the lager lineage based on the mosaic genomes of Saccharomyces bayanus strains NBRC1948 and CBS380T. PLoS One 6:e25821.

33. Brouwers N, Gorter de Vries AR, van den Broek M, Weening SM, Elink Schuurman TD, Kuijpers NGA, Pronk JT, Daran J-MG. 2018. In vivo recombination of Saccharomyces eubayanus maltose-transporter genes yields a chimeric transporter that enables maltotriose fermentation. bioRxiv doi:10.1101/428839.

34. Brouwers N, Gorter de Vries AR, van den Broek M, Weening SM, Elink Schuurman TD, Kuijpers NGA, Pronk JT, Daran JG. 2019. In vivo recombination of Saccharomyces eubayanus maltose-transporter genes yields a chimeric transporter that enables maltotriose fermentation. PLoS Genet 15:e1007853. 
35. Baker EP, Hittinger CT. 2019. Evolution of a novel chimeric maltotriose transporter in Saccharomyces eubayanus from parent proteins unable to perform this function. PLoS Genet 15:e1007786.

36. Mertens S, Steensels J, Saels V, De Rouck G, Aerts G, Verstrepen KJ. 2015. A large set of newly created interspecific yeast hybrids increases aromatic diversity in lager beers. Appl Environ Microbiol:AEM. 02464-02415.

37. Gorter de Vries A, Voskamp MA, van Aalst ACA, Kristensen LH, Jansen $L$, van den Broek $M$, Salazar AN, Brouwers N, Abeel T, Pronk JT, Daran J-MG. 2018. Laboratory evolution of a Saccharomyces cerevisiae $\times \mathrm{S}$. eubayanus hybrid under simulated lager-brewing conditions: genetic diversity and phenotypic convergence. bioRxiv.

38. Bing J, Han P-J, Liu W-Q, Wang Q-M, Bai F-Y. 2014. Evidence for a Far East Asian origin of lager beer yeast. Curr Biol 24:R380-R381.

39. Peris D, Langdon QK, Moriarty RV, Sylvester K, Bontrager M, Charron G, Leducq J-B, Landry C, Libkind D, Hittinger CT. 2016. Complex ancestries of lager-brewing hybrids were shaped by standing variation in the wild yeast Saccharomyces eubayanus. PLoS Genet 12:20.

40. Darling ACE, Mau B, Blattner FR, Perna NT. 2004. Mauve: multiple alignment of conserved genomic sequence with rearrangements. Genome research 14:1394-1403.

41. Brown CA, Murray AW, Verstrepen KJ. 2010. Rapid expansion and functional divergence of subtelomeric gene families in yeasts. Curr Biol 20:895-903.

42. Moller M, Habig M, Freitag M, Stukenbrock EH. 2018. Extraordinary Genome Instability and Widespread Chromosome Rearrangements During Vegetative Growth. Genetics 210:517529.

43. Gordon JL, Byrne KP, Wolfe KH. 2009. Additions, losses, and rearrangements on the evolutionary route from a reconstructed ancestor to the modern Saccharomyces cerevisiae genome. PLoS Genet 5:e1000485.

44. Marques WL, Mans R, Henderson RK, Marella ER, ter Horst J, de Hulster E, Poolman B, Daran J-M, Pronk JT, Gombert AK. 2018. Combined engineering of disaccharide transport and phosphorolysis for enhanced ATP yield from sucrose fermentation in Saccharomyces cerevisiae. Metabolic engineering 45:121-133.

45. Meurer M, Chevyreva V, Cerulus B, Knop M. 2017. The regulatable MAL32 promoter in Saccharomyces cerevisiae: characteristics and tools to facilitate its use. Yeast 34:39-49.

46. Levine J, Tanouye L, Michels CA. 1992. The UAS(MAL) is a bidirectional promotor element required for the expression of both the MAL61 and MAL62 genes of the Saccharomyces MAL6 locus. Curr Genet 22:181-189.

47. Gorter de Vries AR, Groot PA, Broek M, Daran J-MG. 2017. CRISPR-Cas9 mediated gene deletions in lager yeast Saccharomyces pastorianus. Microb Cell Fact 16:222.

48. Vidgren V, Kankainen M, Londesborough J, Ruohonen L. 2011. Identification of regulatory elements in the AGT1 promoter of ale and lager strains of brewer's yeast. Yeast 28:579-594.

49. Dunn B, Sherlock G. 2008. Reconstruction of the genome origins and evolution of the hybrid lager yeast Saccharomyces pastorianus. Genome Res 18:1610-1623.

50. Okuno M, Kajitani R, Ryusui R, Morimoto H, Kodama Y, Itoh T. 2016. Next-generation sequencing analysis of lager brewing yeast strains reveals the evolutionary history of interspecies hybridization. DNA Res 23:67-80.

51. Monerawela C, Bond U. 2017. Brewing up a storm: The genomes of lager yeasts and how they evolved. Biotechnology Advances 35:512-519.

52. Alsammar HF, Naseeb S, Brancia LB, Gilman RT, Wang P, Delneri D. 2019. Targeted metagenomics approach to capture the biodiversity of Saccharomyces genus in wild environments. Environmental Microbiology Reports 11:206-214.

53. Vidgren V, Ruohonen L, Londesborough J. 2005. Characterization and functional analysis of the MAL and MPH Loci for maltose utilization in some ale and lager yeast strains. Appl Environ Microbiol 71:7846-7857. 
807 54. Gallone B, Steensels J, Prahl T, Soriaga L, Saels V, Herrera-Malaver B, Merlevede A, Roncoroni M, Voordeckers K, Miraglia L, Teiling C, Steffy B, Taylor M, Schwartz A, Richardson T, White C, Baele G, Maere S, Verstrepen KJ. 2016. Domestication and Divergence of Saccharomyces cerevisiae Beer Yeasts. Cell 166:1397-1410 e1316.

55. Gibson BR, Storgårds E, Krogerus K, Vidgren V. 2013. Comparative physiology and fermentation performance of Saaz and Frohberg lager yeast strains and the parental species Saccharomyces eubayanus. Yeast 30:255-266.

56. Salazar AN, Gorter de Vries AR, van den Broek $M$, Brouwers $N$, de la Torre Cortès $P$, Kuijpers NGA, Daran J-MG, Abeel T. 2019. Nanopore sequencing and comparative genome analysis confirm lager-brewing yeasts originated from a single hybridization. bioRxiv doi:10.1101/603480:603480.

57. Lei H, Zhao H, Yu Z, Zhao M. 2012. Effects of wort gravity and nitrogen level on fermentation performance of brewer's yeast and the formation of flavor volatiles. Appl Biochem Biotechnol 166:1562-1574.

58. Papapetridis I, Verhoeven MD, Wiersma SJ, Goudriaan M, van Maris AJA, Pronk JT. 2018. Laboratory evolution for forced glucose-xylose co-consumption enables identification of mutations that improve mixed-sugar fermentation by xylose-fermenting Saccharomyces cerevisiae. FEMS Yeast Research 18.

59. Wisselink HW, Toirkens MJ, Wu Q, Pronk JT, van Maris AJA. 2009. Novel evolutionary engineering approach for accelerated utilization of glucose, xylose, and arabinose mixtures by engineered Saccharomyces cerevisiae strains. Appl Environ Microbiol 75:907-914.

60. Bolat I, Romagnoli G, Zhu F, Pronk JT, Daran JM. 2013. Functional analysis and transcriptional regulation of two orthologs of ARO10, encoding broad-substrate-specificity 2oxo-acid decarboxylases, in the brewing yeast Saccharomyces pastorianus CBS1483. FEMS Yeast Res 13:505-517.

61. Verduyn C, Postma E, Scheffers WA, Van Dijken JP. 1992. Effect of benzoic acid on metabolic fluxes in yeasts: a continuous-culture study on the regulation of respiration and alcoholic fermentation. Yeast 8:501-517.

62. Pronk JT. 2002. Auxotrophic Yeast Strains in Fundamental and Applied Research. Applied and Environmental Microbiology 68:2095.

63. Solis-Escalante D, Kuijpers NG, Nadine B, Bolat I, Bosman L, Pronk JT, Daran J-MG, Pascale D-L. 2013. amdSYM, a new dominant recyclable marker cassette for Saccharomyces cerevisiae. FEMS Yeast Res 13:126-139.

64. Marques WL, Mans R, Marella ER, Cordeiro RL, van den Broek M, Daran J-MG, Pronk JT, Gombert AK, van Maris AJA. 2017. Elimination of sucrose transport and hydrolysis in Saccharomyces cerevisiae: a platform strain for engineering sucrose metabolism. FEMS Yeast Res 17:fox006.

65. de Kok S, Yilmaz D, Suir E, Pronk JT, Daran J-MG, van Maris AJA. 2011. Increasing freeenergy (ATP) conservation in maltose-grown Saccharomyces cerevisiae by expression of a heterologous maltose phosphorylase. Metab Eng 13:518-526.

66. Lee ME, DeLoache WC, Cervantes B, Dueber JE. 2015. A Highly Characterized Yeast Toolkit for Modular, Multipart Assembly. ACS Synth Biol 4:975-986.

67. Gao Y, Zhao Y. 2014. Self-processing of ribozyme-flanked RNAs into guide RNAs in vitro and in vivo for CRISPR-mediated genome editing. Journal of integrative plant biology 56:343-349.

68. Juergens H, Varela JA, Gorter de Vries AR, Perli T, Gast VJ, Gyurchev NY, Rajkumar AS, Mans R, Pronk JT, Morrissey JP. 2018. Genome editing in Kluyveromyces and Ogataea yeasts using a broad-host-range Cas9/gRNA co-expression plasmid. FEMS yeast research 18:foy012.

69. Engler C, Kandzia R, Marillonnet S. 2008. A One Pot, One Step, Precision Cloning Method with High Throughput Capability. PLOS ONE 3:e3647. 
70. van Rossum HM, Kozak BU, Niemeijer MS, Duine HJ, Luttik MA, Boer VM, Kötter P, Daran JMG, van Maris AJA, Pronk JT. 2016. Alternative reactions at the interface of glycolysis and citric acid cycle in Saccharomyces cerevisiae. FEMS Yeast Res 16.

71. Gietz RD, Schiestl RH. 2007. High-efficiency yeast transformation using the LiAc/SS carrier DNA/PEG method. Nature protocols 2:31.

72. Pengelly RJ, Wheals AE. 2013. Rapid identification of Saccharomyces eubayanus and its hybrids. FEMS Yeast Res 13:156-161.

73. Koren S, Walenz BP, Berlin K, Miller JR, Bergman NH, Phillippy AM. 2017. Canu: scalable and accurate long-read assembly via adaptive k-mer weighting and repeat separation. Genome Res:gr. 215087.215116.

74. Walker BJ, Abeel T, Shea T, Priest M, Abouelliel A, Sakthikumar S, Cuomo CA, Zeng Q, Wortman J, Young SK. 2014. Pilon: an integrated tool for comprehensive microbial variant detection and genome assembly improvement. PloS one 9:e112963.

75. Li H, Durbin R. 2010. Fast and accurate long-read alignment with Burrows-Wheeler transform. Bioinformatics 26:589-595.

76. Holt C, Yandell M. 2011. MAKER2: an annotation pipeline and genome-database management tool for second-generation genome projects. BMC bioinformatics 12:491.

77. Korf I. 2004. Gene finding in novel genomes. BMC bioinformatics 5:59.

78. Stanke M, Keller O, Gunduz I, Hayes A, Waack S, Morgenstern B. 2006. AUGUSTUS: ab initio prediction of alternative transcripts. Nucleic acids research 34:W435-W439.

79. Camacho C, Coulouris G, Avagyan V, Ma N, Papadopoulos J, Bealer K, Madden TL. 2009. BLAST+: architecture and applications. BMC bioinformatics 10:421.

80. Tai SL, Boer VM, Daran-Lapujade P, Walsh MC, de Winde JH, Daran J-M, Pronk JT. 2005. Two-dimensional transcriptome analysis in chemostat cultures combinatorial effects of oxygen availability and macronutrient limitation in Saccharomyces cerevisiae. Journal of Biological Chemistry 280:437-447.

81. Dobin A, Gingeras TR. 2016. Optimizing RNA-Seq mapping with STAR, p 245-262, Data Mining Techniques for the Life Sciences. Springer.

82. Anders S, PyI PT, Huber W. 2015. HTSeq-a Python framework to work with high-throughput sequencing data. Bioinformatics 31:166-169.

83. Robinson MD, McCarthy DJ, Smyth GK. 2010. edgeR: a Bioconductor package for differential expression analysis of digital gene expression data. Bioinformatics 26:139-140.

84. McCarthy DJ, Chen Y, Smyth GK. 2012. Differential expression analysis of multifactor RNASeq experiments with respect to biological variation. Nucleic acids research 40:4288-4297.

85. Anders S, McCarthy DJ, Chen Y, Okoniewski M, Smyth GK, Huber W, Robinson MD. 2013. Count-based differential expression analysis of RNA sequencing data using R and Bioconductor. Nature protocols 8:1765.

86. Entian K-D, Kötter P. 2007. 25 Yeast genetic strain and plasmid collections. Methods in Microbiology 36:629-666. 
bioRxiv preprint doi: https://doi.org/10.1101/679563; this version posted June 28, 2019. The copyright holder for this preprint (which was not certified by peer review) is the author/funder, who has granted bioRxiv a license to display the preprint in perpetuity. It is made available under aCC-BY-NC-ND 4.0 International license.

Table 1: Saccharomyces strains used in this study. The abbreviation mal indicates mal11-mal12::loxP mal21-mal22::loxP mal31-32::IoxP. Mal and Mtt denote the maltose and maltotriose phenotype respectively.

\begin{tabular}{|c|c|c|c|}
\hline Name & Species & Relevant genotype & Origin \\
\hline CDFM21L.1 & S. eubayanus & Wildtype $\mathrm{Mal}^{-} \mathrm{Mtt}^{-}$ & (38) \\
\hline ABFM5L.1 & S. eubayanus & Wildtype Mal' Mtt ${ }^{-}$ & (38) \\
\hline CBS 12357 & S. eubayanus & Wildtype $\mathrm{Mal}^{+} \mathrm{Mtt}^{-}$ & (1) Westerdijk institute \\
\hline CBS 1483 & S. pastorianus & Wildtype $\mathrm{Mal}^{+} \mathrm{Mtt}^{+}$ & Westerdijk institute \\
\hline CEN.PK113-7D & S. cerevisiae & $\begin{array}{l}\text { MATa MAL1 } \times \text { MAL2x MAL3x MAL4x MAL2-8C SUC2 LEU2 } \\
\text { URA3 }\end{array}$ & $(86)$ \\
\hline IMZ616 & S. cerevisiae & 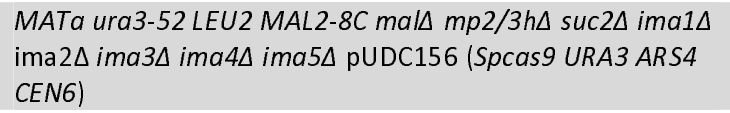 & (44) \\
\hline IMX1365 & S. cerevisiae & 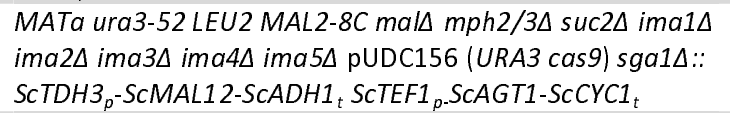 & (13) \\
\hline IMX1702 & S. cerevisiae & 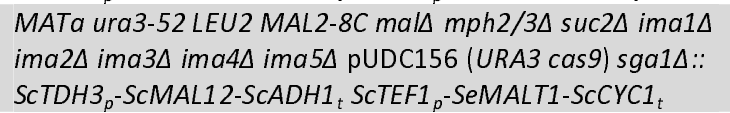 & This study \\
\hline IMX1704 & S. cerevisiae & 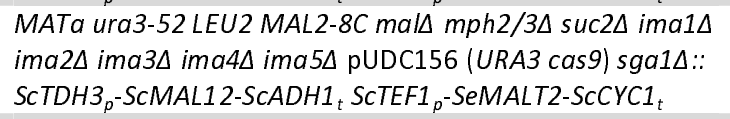 & This study \\
\hline IMX1706 & S. cerevisiae & 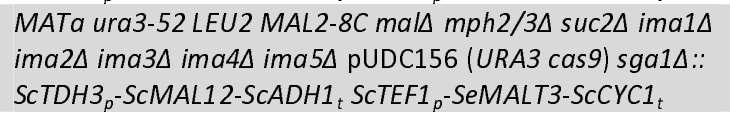 & This study \\
\hline $\mathrm{IMX} 1708$ & S. cerevisiae & 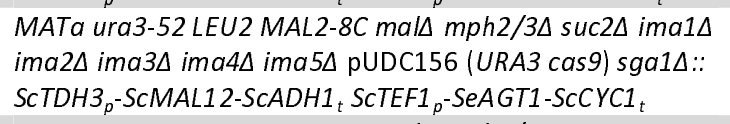 & This study \\
\hline IMX1313 & S. cerevisiae & 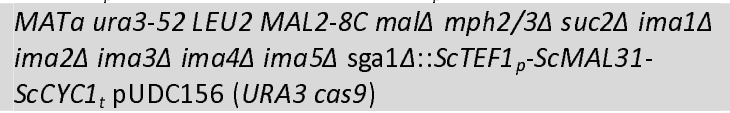 & This study \\
\hline IMX1313 $\Delta$ & S. cerevisiae & 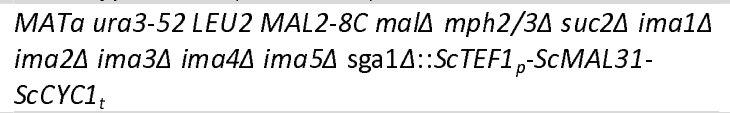 & This study \\
\hline IMZ752 & S. cerevisiae & 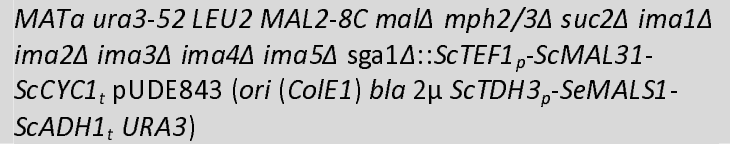 & This study \\
\hline IMZ753 & S. cerevisiae & 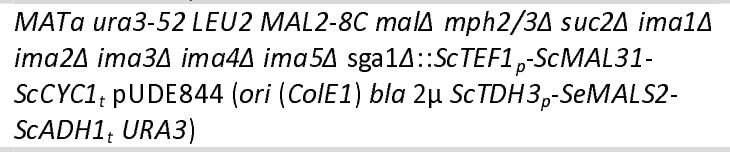 & This study \\
\hline IMK820 & S. eubayanus & MATa/MAT $\alpha$ Semalt1 $\Delta /$ Semalt1 $\Delta$ & This study \\
\hline IMK823 & S. eubayanus & 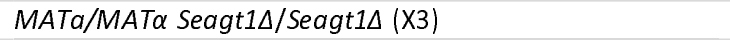 & This study \\
\hline IMX1939 & S. eubayanus & 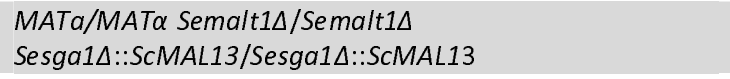 & This study \\
\hline IMX1940 & S. eubayanus & 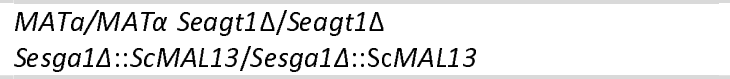 & This study \\
\hline IMX1762 & S. eubayanus & 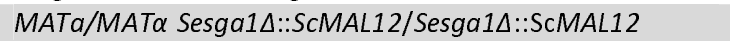 & This study \\
\hline IMX1765 & S. eubayanus & 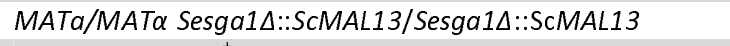 & This study \\
\hline CBC-1 & S. cerevisiae & MATa/MAT $\alpha \mathrm{Mal}^{+} \mathrm{Mtt}^{-}$ & Lallemand \\
\hline HTSH020 & $\begin{array}{l}\text { S. cerevisiae XS. } \\
\text { eubayanus }\end{array}$ & $\mathrm{MATa} / \mathrm{MAT} \alpha \mathrm{Mal}^{+} \mathrm{Mtt}^{+}$ & This study \\
\hline
\end{tabular}

\footnotetext{
${ }^{a}$ Westerdijk Fungal Biodiversity Institute www.westerdijkinstitute.nl/
} 
bioRxiv preprint doi: https://doi.org/10.1101/679563; this version posted June 28,2019 . The copyright holder for this preprint (which was not certified by peer review) is the author/funder, who has granted bioRxiv a license to display the preprint in perpetuity. It is made available under

\begin{tabular}{|c|c|c|}
\hline Plasmid & Relevant genotype & Origin \\
\hline p426-TEF-amdS & ori (ColE1) bla $2 \mu$ amdSYM TEF1 $1_{p r} C Y C 1_{\text {ter }}$ & (64) \\
\hline pUD444 & ori (ColE1) bla $2 \mu$ amdSYM ScTEF $1_{p r}$-ScMAL31-ScCYC1 $1_{\text {ter }}$ & This study \\
\hline pUD794 & ori (ColE1) bla $2 \mu$ amdSYM ScTEF $1_{p r}$-SeMALT1-ScCYC1 $1_{\text {ter }}$ & This study \\
\hline pUD795 & ori (ColE1) bla $2 \mu$ amdSYM ScTEF $1_{p r}$-SeMALT2-ScCYC1 $1_{\text {ter }}$ & This study \\
\hline pUD796 & ori (ColE1) bla $2 \mu$ amdSYM ScTEF1 $1_{p r}$ SeMALT3-ScCYC1 $1_{\text {ter }}$ & This study \\
\hline pUD797 & ori (ColE1) bla $2 \mu$ amdSYM ScTEF $1_{p r}$-SeAGT1-ScCYC1 $1_{\text {ter }}$ & This study \\
\hline pUDE044 & ori (ColE1) bla $2 \mu$ ScTDH $3_{p r}-S C M A L 12-S c A D H 1_{\text {ter }}$ URA3 & (65) \\
\hline pUDE843 & ori (ColE1) bla $2 \mu S c T D H 3_{p r}$-SeMALS1-SCADH1 $1_{\text {ter }}$ URA3 & This study \\
\hline pUDE844 & ori (ColE1) bla $2 \mu$ ScTDH $3_{p r}$-SeMALS2-SCADH1 $1_{\text {ter }}$ URA 3 & This study \\
\hline pUDE780 & ori (ColE1) bla $2 \mu$ ScPGK1 $1_{p r}-S c M A L 13-S c T E F 2_{\text {ter }}$ URA 3 & This study \\
\hline pUDP002 & $\begin{array}{l}\text { ori (ColE1) bla panARSopt Hyg ScTDH } 3_{p r} \text {-Bsal-Bsal-ScCYC1 } 1_{t e r} \\
\text { AaTEF1 }_{p r}-S p c a s 9^{\mathrm{D} 147 Y \mathrm{PA} 11 \mathrm{~T}}-\mathrm{SCPHO} 5_{t e r}\end{array}$ & (68) \\
\hline pUDP004 & $\begin{array}{l}\text { ori (ColE1) bla panARSopt amdSYM ScTDH } 3_{p r} \text {-Bsal-Bsal-ScCYC1 } 1_{t e r} \\
\text { AaTEF1 } 1_{p r}-S p c a s 9^{D 147 Y P A 11 T}-S c P H O 5_{t e r}\end{array}$ & (47) \\
\hline pUDP052 & 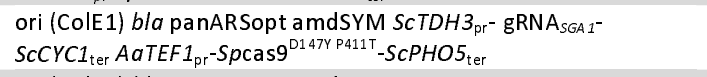 & (13) \\
\hline pUDP091 & $\begin{array}{l}\text { ori (ColE1) bla panARSopt amdSYM SCTDH } 3_{\mathrm{pr}} \text { - } \text { gRNA }_{\text {SeMALT1 }} \\
\text { ScCYC1 } \\
\text { ter } A a T E F 1_{\text {pr }}-S p c a s 9^{\text {D14TYPA11T }}-S c P H O 5_{\text {ter }}\end{array}$ & This study \\
\hline pUDP090 & 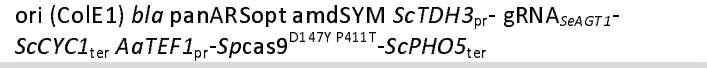 & This study \\
\hline pUDR119 & 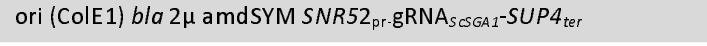 & (70) \\
\hline pYTK074 & ori (ColE1) cat URA3 & (66) \\
\hline pYTK082 & cat $2 \mu$ & (66) \\
\hline pYTK083 & ori (ColE1) bla & (66) \\
\hline pUD631 & ori (ColE1) bla gRNA SEMALT1 & (13) \\
\hline pUD634 & ori (ColE1) bla gRNA $\mathrm{S}_{S E A T T 1}$ & This study \\
\hline pUDC156 & ori (ColE1) bla ARS4 CEN6 URA3 Spcas9 & (44) \\
\hline
\end{tabular}




\section{Legends to Figures}

905 Figure 1. Genome comparison between CDFM21L.1 and CBS 12357 ${ }^{\top}$. (A) Translocations in

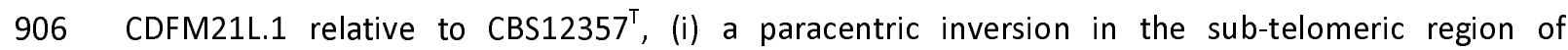
907 chromosome VII, involving approximately $8 \mathrm{kbp}$, (ii) a translocation of approximately $12 \mathrm{kbp}$ from the 908 left sub-telomeric region of chromosome VIII to the right sub-telomeric region of chromosome VI, 909 and (iii) a reciprocal translocation between approximately $20 \mathrm{kbp}$ from the right sub-telomeric region 910 of chromosome V and approximately $60 \mathrm{kbp}$ from the center of chromosome XII.Red lines depicted chromosomes separation. Genome syntheny is indicated with colored blocks. (B) Relative chromosome position of gene presence differences between CDFM21L.1 (blue) and CBS $12357^{\top}$ (red). (C) Representation of the assembled CDFM21L.1S. eubayanus chromosomes, the black boxes denote newly added sequences. New annotated open reading frames and gene entries modified relative to the CBS 12357T draft genome (13).

Figure 2. Organization of sub-telomeric regions involving MAL genes and SeAGT1 in CDFM21L.1. (A) Chromosome sections are represented as lines and red boxes denote telomeres. CDFM21L.1 genome harbors three SeMALT genes of which SeMALT2 and SeMALT3 have a mutation resulting in an early stop codon and truncated protein (denoted with *). Three copies of SeAGT1 were found close to the

921 telomeres on CHRVII, XIV and XV. Furthermore there are two intact SeMALS genes on CHRII (1) and

922 XII (2) and three SeMALR genes on CHRV (1) and XIII (2) whose copy on CHRV is also mutated 923 (SeMALR1*). The gene and interval sizes are approximately to scale. (B) Phylogeny of Saccharomyces

924 SeAGT1 genes described in S. cerevisiae, S. eubayan us, and lager-brewing hybrid S. pastorian us. (C)

925 Nucleotide percentage identities between AGT1 orthologs from S. cerevisiae, S. eubayanus, and 926 lager-brewing hybrid S. pastorianus. Green color indicates highest similarity between SeAGT1 and 927 SeAGT1 genes from S. pastorianus strains CBS 1483 and WS3470. Red color indicates similarity 928 between SeAGT1 from North American strains with SeAGT1 genes from Asian S. eubayanus and S. pastorianus strains CBS 1483 and WS3470. 
931 Figure 3. Characterization of sugar consumption of S. pastorianus CBS1483 (black) and S.

932 eubayanus CBS $12357^{\top}$ (blue), CDFM21L.1 (red), and ABFM5L.1 (green) on wort. For every sample,

933 glucose $(\boldsymbol{\nabla})$, maltose $(\mathbf{A})$, maltotriose $(\boldsymbol{\nabla})$ and ethanol $(\boldsymbol{)})$ were measured from the supernatant.

934 Strains were grown at $12{ }^{\circ} \mathrm{C}$ for 110 hours in infusion Neubor flasks. Samples were filtered through a

$9350.22 \mu \mathrm{m}$ filter and analyzed on HPLC. Data represents average and standard deviation of three 936 biological replicates.

937

940

941

942

943

944

945

946

947

948

949

950

951

952

953

954

Figure 4. Overexpression of SeMALT, SeAGT1 and SeMALS genes in a maltose negative background S. cerevisiae strain. Maltose negative background strain IMZ616 (ש), IMX1365 overexpressing

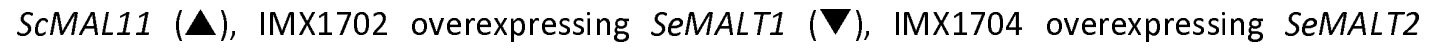
IMX1706 overexpressing SeMALT3 (O) and IMX1708 overexpressing SeAGT1 ( $\square$ ) were grown on SM $2 \%$ maltose or maltotriose at $20{ }^{\circ} \mathrm{C}$. Growth on maltose (A) and on maltotriose (B) was monitored based on optical density $\left(O D_{660 \mathrm{~nm}}\right)$ and concentrations of maltose and maltotriose in culture supernatants were measured by HPLC. Data are presented as average and standard deviation of two biological replicates. (C) IMX1313 overexpressing only SCMAL31 (O), IMZ752 overexpressing SCMAL31 and SeMALS1 $(\triangle)$ and IMZ753 overexpressing SCMAL31 and SeMALS2 ( $\nabla)$ grown on SM maltose $2 \%$. Growth was monitored based on optical density measurement at $660 \mathrm{~nm}\left(\mathrm{OD}_{660 \mathrm{~nm}}\right)$ and maltose in culture supernatants was measured by HPLC. Data represents average and standard deviation of two biological replicates.

Figure 5. Integration of SCMAL13 in CDFM21L.1 restores growth on maltose and maltotriose and enables native SeMALT1 and SeAGT1 characterization in knockout strains IMK820 and IMK823. (A) Characterization of S. eubayanus IMX1765 overexpressing SCMAL13 ( $\nabla$ ) and CDFM21L.1 ( $\mathbf{\square})$ on SM with glucose, maltose or maltotriose at $20^{\circ} \mathrm{C} . \mathrm{OD}_{660 \mathrm{~nm}}$ was measured (black) and sugar (black) and ethanol (red) concentrations were determined from the supernatant by HPLC. (B) Overview of 
955

956

957

958

959

960

961

962

963

964

965

966

967

968

969

970

971

972

973

974

975

976

977

constructed knockout strains. Knockouts of SeMALT1 (IMK820) and SeAGT1 (IMK823) were made

with CRISPR-Cas9. Subsequently the SeSGA1 locus was replaced by SCPGK1 $1_{p}-S C M A L 13-S C T E F 2_{t}$ using

CRISPR-Cas9 in both strains resulting in IMX1939 and IMX1940, respectively. (C) S. eubayanus strains

IMK820 (四), IMK823 ( $\mathbf{\Delta})$, IMX1939 ( $\boldsymbol{\nabla}$ ) and IMX1940 ( $)$ were characterized on SM with maltose or

maltotriose at $20^{\circ} \mathrm{C} . \mathrm{OD}_{660 \mathrm{~nm}}$ was measured (black) and sugar (black) and ethanol (red)

concentrations were determined from the supernatant by HPLC. All data represents average and

standard deviation of biological duplicates.

Figure 6. Expression levels of maltose metabolism genes in IMX1765. Normalized transcript levels of maltose metabolism genes from IMX1765mid-exponential phase grown on glucose (red), maltose (blue)and maltotriose (green) at $20^{\circ} \mathrm{C}$ were calculated from duplicate RNA sequencing experiments (2x $150 \mathrm{bp}$ ) using the FPKM method. All data represents average and standard deviation of two biological duplicates.

Figure 7. Hybridization of maltotriose deficient S. cerevisiae and S. eubayanus leading to crosstalk restoring maltotriose utilization, explains S. pastorianus phenotype. (A) Characterization of $S$.

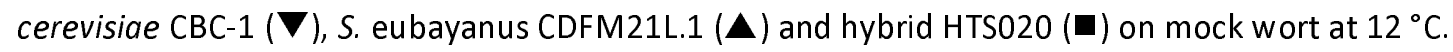
Consumption of maltose (red), maltotriose (blue) and production of ethanol (green) was measured from supernatant by HPLC. Data represents average and standard deviation from biological triplicates. (B) Normalized transcript levels of maltose metabolism genes from HTSO20 midexponential phase grown on glucose (red), maltose (blue)and maltotriose (green) at $20^{\circ} \mathrm{C}$ were calculated from duplicate RNA sequencing experiments (2x $150 \mathrm{bp}$ ) using the FPKM method. All data represents average and standard deviation of two biological duplicates. 


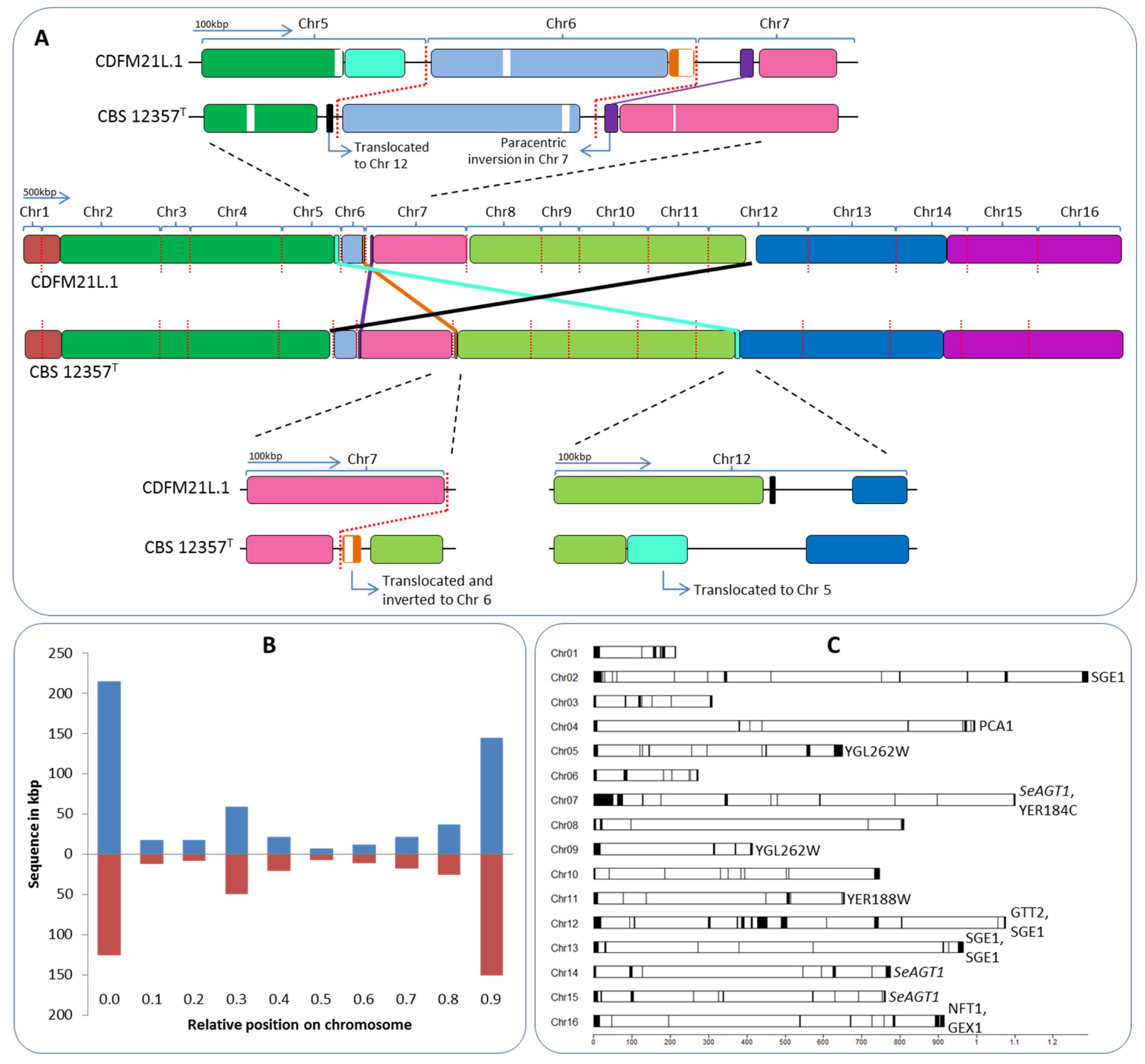




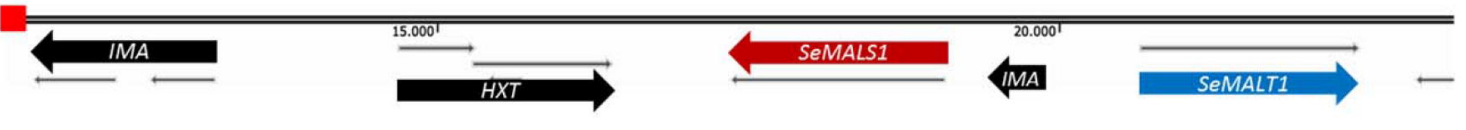

CHRV

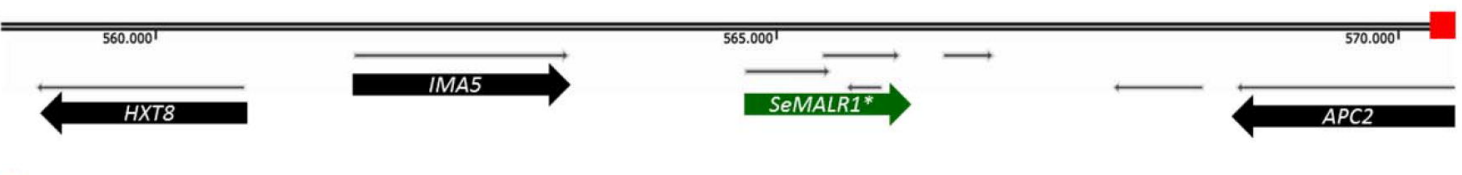

CHRVII

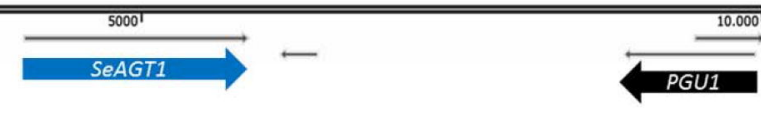

CHRXI

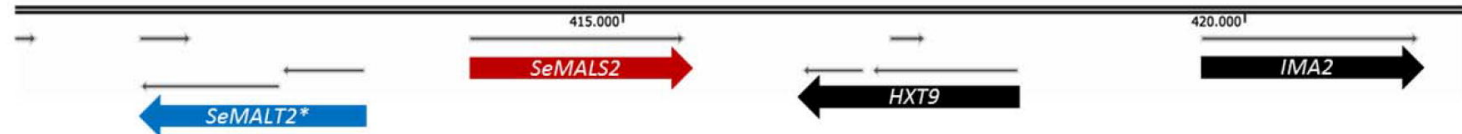

CHRXIII

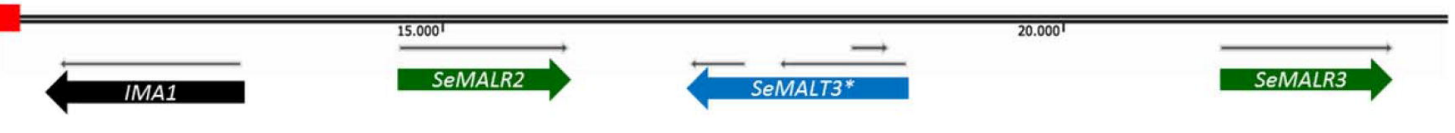

CHRXIV

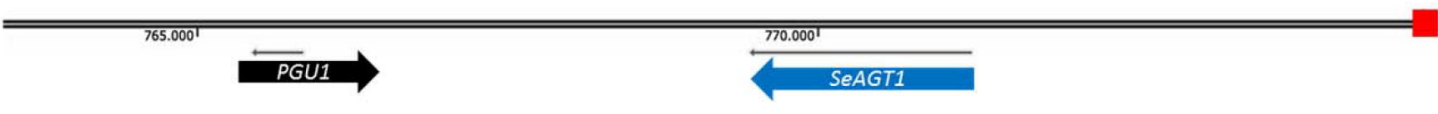

CHRXV

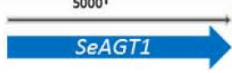

8.26

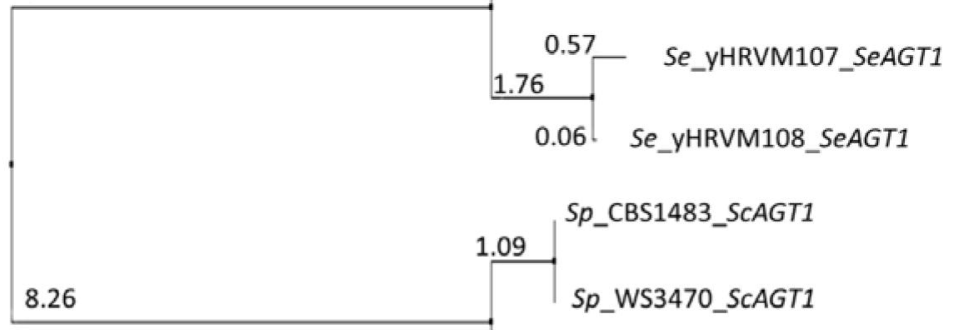

Sc_S288C_SCAGT1 Sp_WS3470_SCAGT1 SP_CBS1483_SCAGT1

Se_CDFM21L.1_SEAGT1 $S p \_W S 3470 \_S e A G T 1$ $S p \_C B S 1483$ SeAGT1 Se_yHRVM107_SeAGT1 Se_YHRVM108_SeAGT1
0.21 SC_S288C_SCAGT1
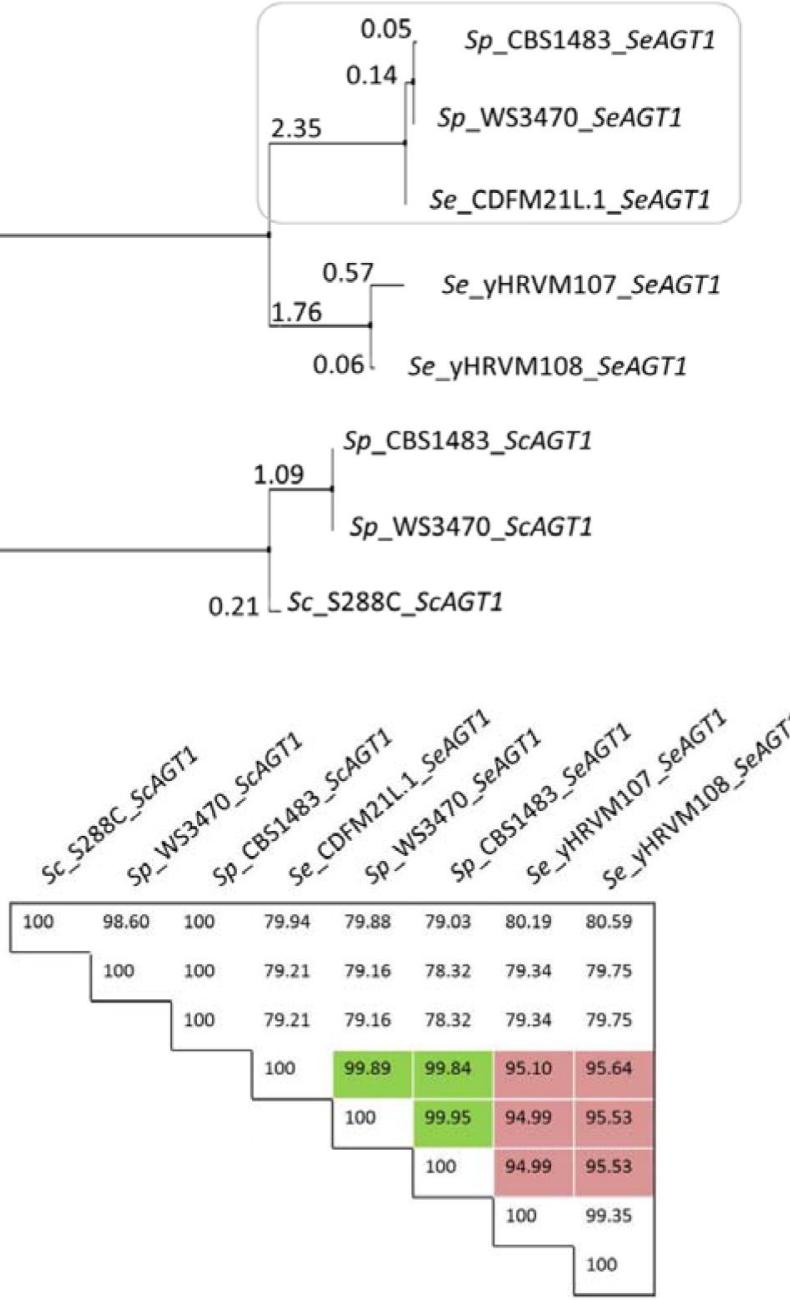

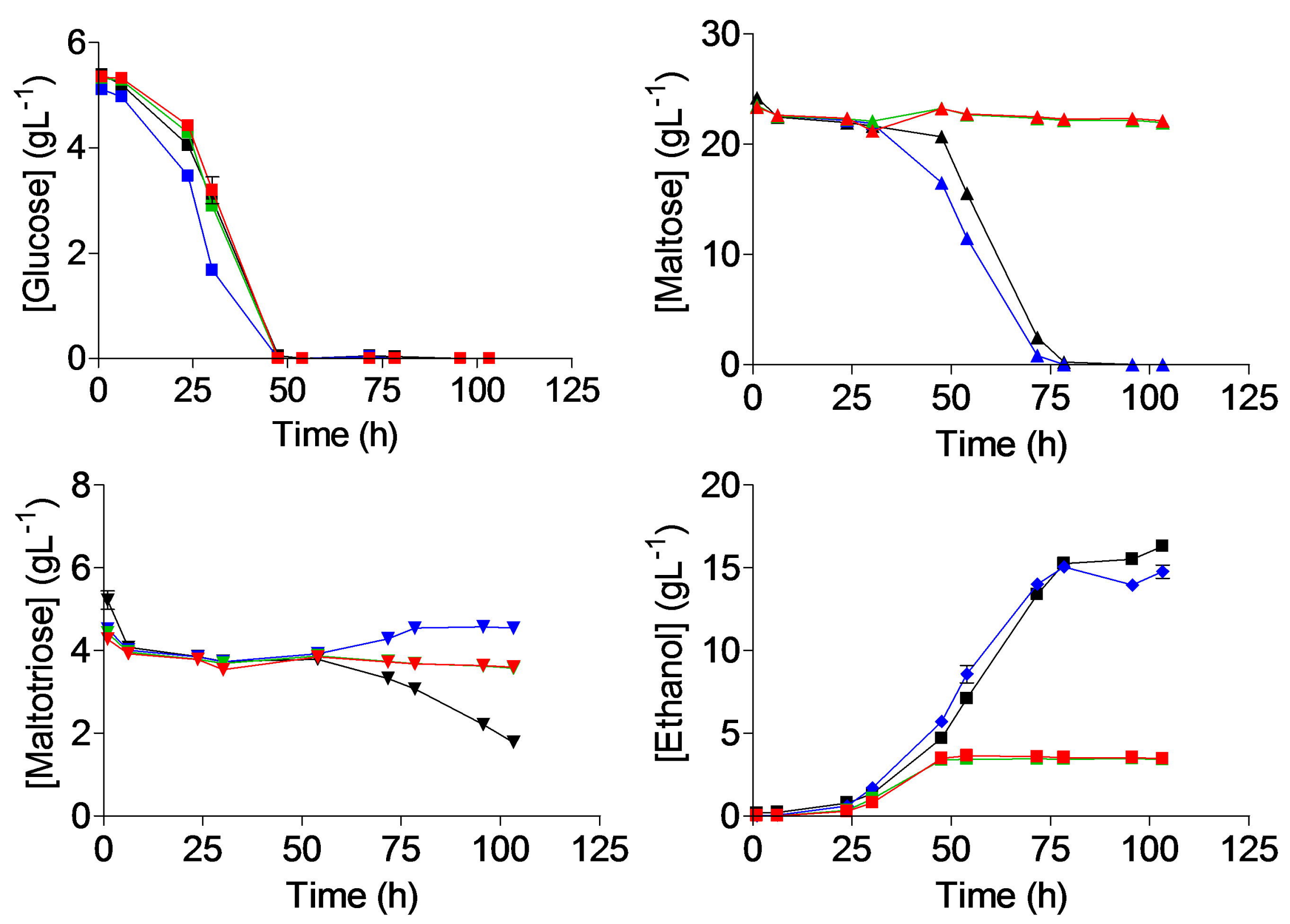

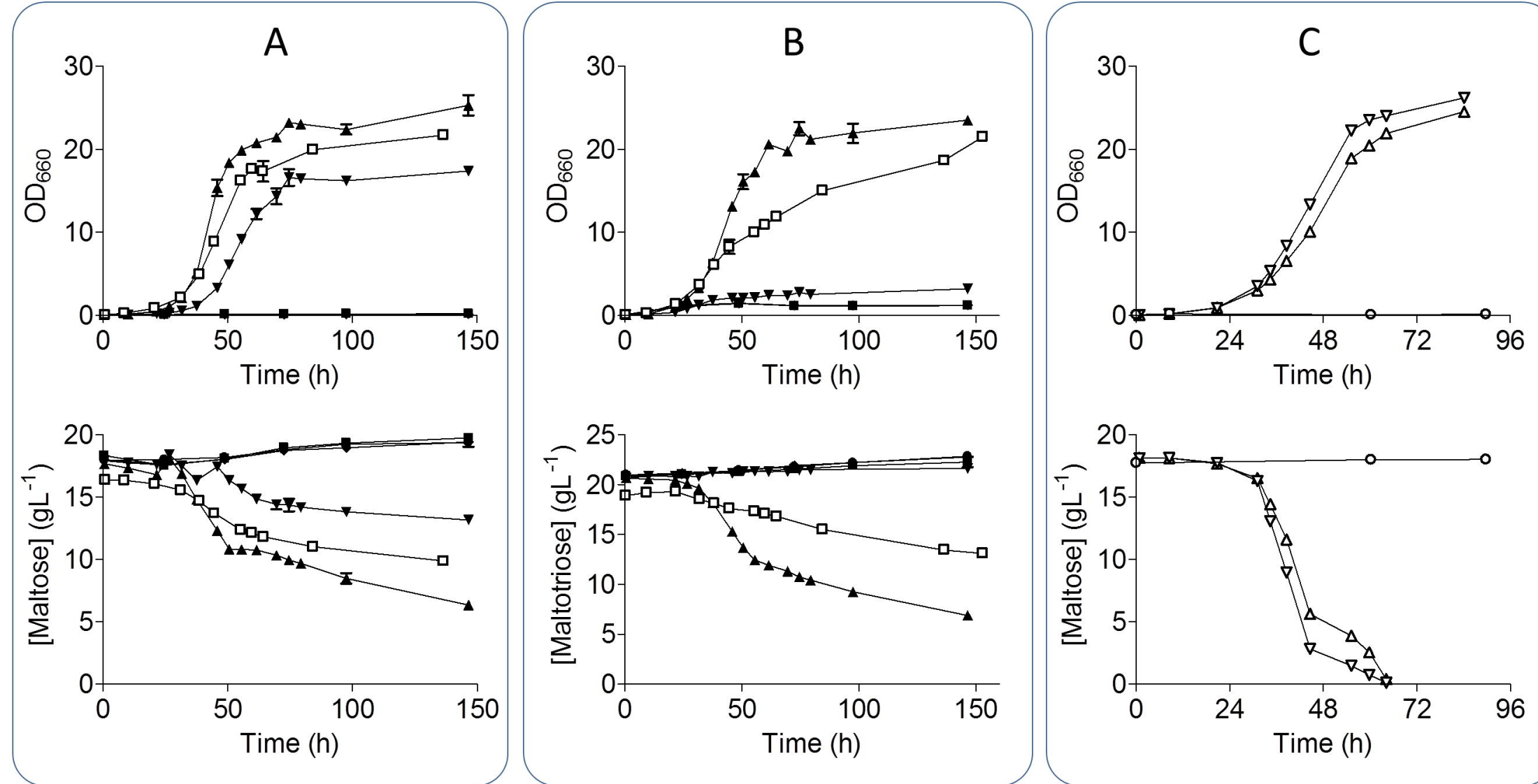


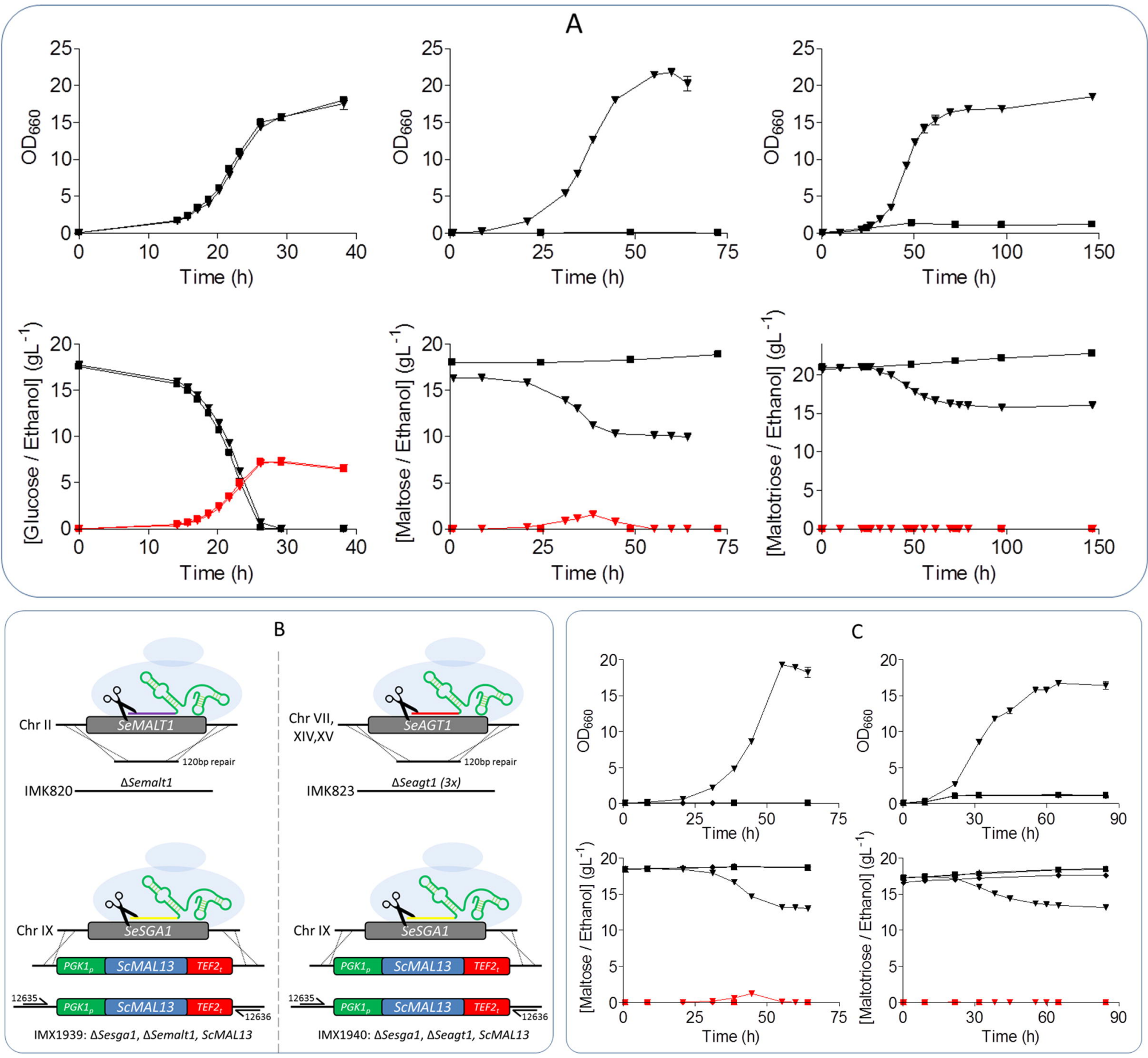




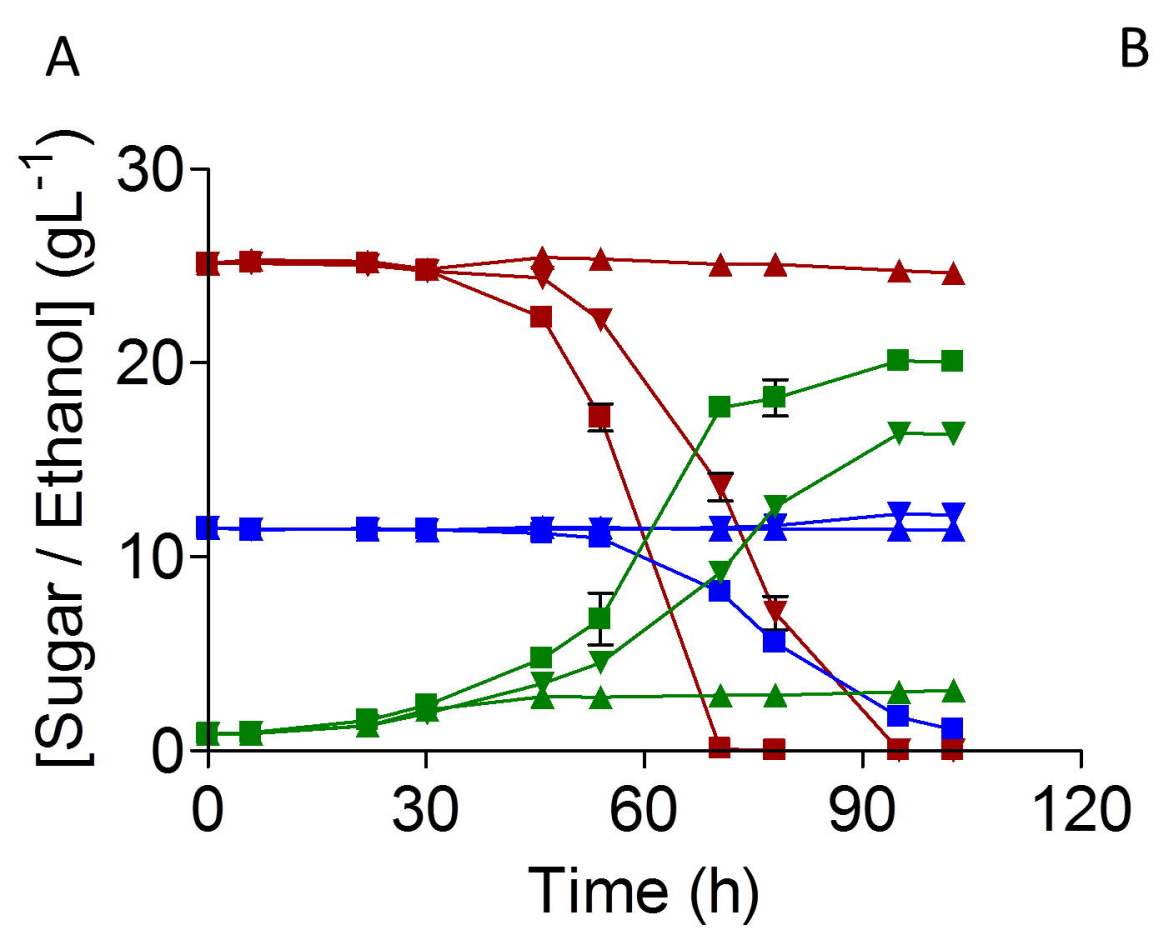

B

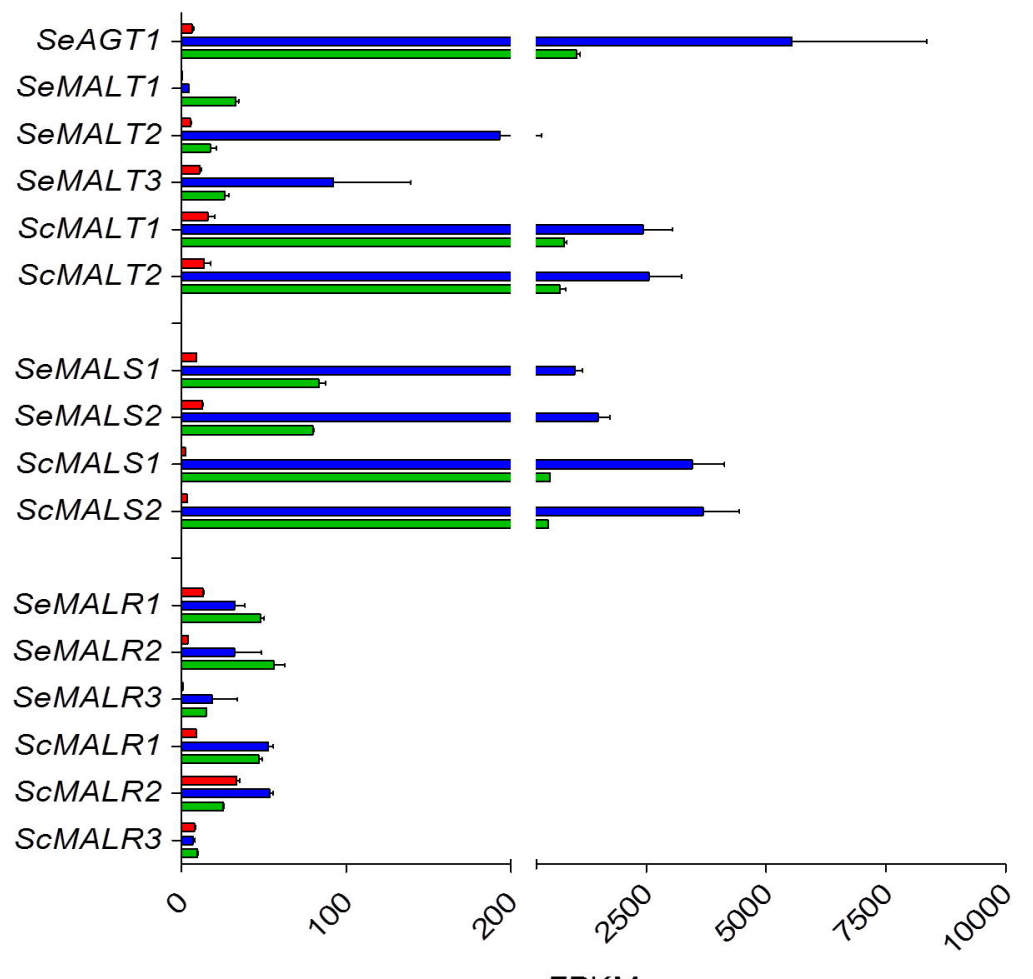

